\title{
A DIRECT COUPLING OF LOCAL DISCONTINUOUS GALERKIN AND BOUNDARY ELEMENT METHODS
}

\author{
GABRIEL N. GATICA, NORBERT HEUER, AND FRANCISCO-JAVIER SAYAS
}

\begin{abstract}
The coupling of local discontinuous Galerkin (LDG) and boundary element methods (BEM), which has been developed recently to solve linear and nonlinear exterior transmission problems, employs a mortar-type auxiliary unknown to deal with the weak continuity of the traces at the interface boundary. As a consequence, the main features of LDG and BEM are maintained and hence the coupled approach benefits from the advantages of both methods. In this paper we propose and analyze a simplified procedure that avoids the mortar variable by employing LDG subspaces whose functions are continuous on the coupling boundary. The continuity can be implemented either directly or indirectly via the use of Lagrangian multipliers. In this way, the normal derivative becomes the only boundary unknown, and hence the total number of unknown functions is reduced by two. We prove the stability of the new discrete scheme and derive an a priori error estimate in the energy norm. A numerical example confirming the theoretical result is provided. The analysis is also extended to the case of nonlinear problems and to the coupling with other discontinuous Galerkin methods.
\end{abstract}

\section{INTRODUCTION}

The coupling of local discontinuous Galerkin and boundary element methods, as applied to linear exterior boundary value problems in the plane, has been introduced and analyzed for the first time in [18. The model problem there is the Poisson equation in an annular domain coupled with the Laplace equation in the surrounding unbounded exterior region. The corresponding extension to a class of nonlinear-linear exterior transmission problems, which is also motivated by previous applications of the LDG method to some nonlinear problems in heat conduction and fluid mechanics (see, e.g. 7], 8], and 23]), was developed recently in 9], [10, and [1]. In these works, the authors consider a nonlinear elliptic equation in divergence form in an annular region coupled with discontinuous transmission conditions on the interface boundary and the Poisson equation in the exterior unbounded domain. In both the linear and nonlinear cases the technique employed resembles the usual coupling of finite element and boundary element methods, but

Received by the editor November 1, 2007 and, in revised form, April 29, 2009.

2000 Mathematics Subject Classification. Primary 65N30, 65N38, 65N12, 65 N15.

Key words and phrases. Boundary elements, local discontinuous Galerkin method, coupling, error estimates.

This research was partially supported by FONDAP and BASAL projects CMM, Universidad de Chile, by Centro de Investigación en Ingeniería Matemática $\left(\mathrm{CI}^{2} \mathrm{MA}\right)$, Universidad de Concepción, by FONDECYT project no. 1080044, by Spanish FEDER/MCYT Project MTM2007-63204, and by Gobierno de Aragón (Grupo Consolidado PDIE).

(C)2010 American Mathematical Society Reverts to public domain 28 years from publication 
the corresponding analysis becomes quite different. In particular, in order to deal with the weak continuity of the traces at the coupling boundary, a mortar-type auxiliary unknown representing an interior approximation of the normal derivative needs to be defined. Hence, different mesh sizes on that boundary and special relationships between them are required. In addition, the continuity and ellipticity estimates of the bilinear form involved hold with different mesh-dependent norms, and Strang-type a priori error estimates instead of the usual Céa ones are obtained.

In the present paper we simplify the approach from 18 and develop a direct procedure for the coupling of LDG and BEM which does not make use of any mortar unknown. Instead, it employs a finite element subspace of functions that are required to be continuous only on the coupling boundary $\Gamma$. Consequently, in this paper, the normal derivative becomes the only boundary unknown and then the total number of unknown functions is reduced by two. The continuity of LDG functions on $\Gamma$ can be implemented directly by considering appropriate LDG subspaces. However, one can maintain the full flexibility of the LDG method by implementing the continuity condition via a Lagrangian multiplier. In this way standard LDG and BEM implementations are sufficient for the coupling procedure.

In order to introduce the model problem let $\Omega_{0}$ be a simply connected and bounded domain in $\mathbb{R}^{2}$ with polygonal boundary $\Gamma_{0}$. Then, given $f \in L^{2}\left(\mathbb{R}^{2} \backslash \bar{\Omega}_{0}\right)$ with compact support, we consider the exterior Dirichlet problem:

$$
\begin{gathered}
-\Delta u=f \quad \text { in } \quad \mathbb{R}^{2} \backslash \bar{\Omega}_{0}, \quad u=0 \quad \text { on } \quad \Gamma_{0}, \\
u(\boldsymbol{x})=\mathcal{O}(1) \quad \text { as } \quad|\boldsymbol{x}| \rightarrow \infty .
\end{gathered}
$$

Next, let $\Gamma$ be a closed polygonal curve such that the support of $f$ is inside the annular domain $\Omega$ enclosed by $\Gamma_{0}$ and $\Gamma$. We assume that this support does not intersect $\Gamma$. Then (1.1) can be written as the Poisson equation in $\Omega$ :

$$
-\Delta u=f \quad \text { in } \Omega, \quad u=0 \quad \text { on } \quad \Gamma_{0},
$$

and the Laplace equation in the exterior domain $\Omega_{e}:=\mathbb{R}^{2} \backslash\left(\bar{\Omega}_{0} \cup \bar{\Omega}\right)$ :

$$
-\Delta u_{e}=0 \quad \text { in } \quad \Omega_{e}, \quad u_{e}(\boldsymbol{x})=\mathcal{O}(1) \quad \text { as } \quad|\boldsymbol{x}| \rightarrow \infty,
$$

coupled by the transmission conditions:

$$
u=u_{e} \quad \text { on } \Gamma \text { and } \partial_{\boldsymbol{\nu}} u=\partial_{\boldsymbol{\nu}} u_{e} \text { on } \Gamma .
$$

Here, $\partial_{\boldsymbol{\nu}} u$ denotes the normal derivative of $u$ with normal vector pointing outside $\Omega$. The purpose of this work is to solve numerically (1.1) by means of a new LDGBEM coupling which, similarly to [18, consists of applying the LDG method to (1.2) and the BEM to (1.3).

The remainder of this work is organized as follows. In Section 2 we introduce the boundary integral equation formulation in $\Omega_{e}$, define the LDG method in $\Omega$, and establish the resulting coupled LDG-BEM approach. Next, in Section 3 we prove the unique solvability and stability of our discrete scheme. The associated a priori error analysis is provided in Section 4. Then, in Section 5 we describe a Lagrange multiplier based implementation of the coupled scheme which maintains the discontinuous character of the LDG method. The good performance of this scheme is illustrated with a simple numerical example, which also confirms the theoretical rate of convergence of the method for the regular case $u \in H^{2}(\Omega)$. In Section $[6$ we extend our analysis to the class of nonlinear problems studied in 9 , 
[10], and [1]. Finally, in Section 7 we discuss some aspects of the coupling of BEM with other discontinuous Galerkin methods.

Throughout this paper, $c$ and $C$ denote positive constants, independent of the parameters and functions involved, which may take different values at different occurrences. Given any linear space $V$, the corresponding vector-valued space $V \times V$ endowed with the product norm will be denoted by $\mathbf{V}$. If $\mathcal{O}$ is an open set, its closure, or a polygonal curve, and $s \in \mathbb{R}$, then $|\cdot|_{s, \mathcal{O}}$ and $\|\cdot\|_{s, \mathcal{O}}$ denote the seminorm and norm in the Sobolev space $H^{s}(\mathcal{O})$. In particular, the norms of $H^{s}(\Gamma)$ are denoted by $\|\cdot\|_{s, \Gamma}$. Also, $\langle\cdot, \cdot\rangle$ denotes both the $L^{2}(\Gamma)$ inner product and its extension to the duality pairing of $H^{-s}(\Gamma) \times H^{s}(\Gamma)$.

\section{The COUPLED LDG-BEM APPROACH}

2.1. The boundary integral formulation in the exterior domain. We use Green's representation formula for $u_{e}$ in $\Omega_{e}$,

$$
u_{e}(\mathbf{x})=\int_{\Gamma} \partial_{\nu(\boldsymbol{y})} E(\mathbf{x}, \boldsymbol{y}) u(\boldsymbol{y}) d s_{\boldsymbol{y}}-\int_{\Gamma} E(\mathbf{x}, \boldsymbol{y}) \lambda(\boldsymbol{y}) d s_{\boldsymbol{y}}+c \quad \forall \mathbf{x} \in \Omega_{e},
$$

where $E(\boldsymbol{x}, \boldsymbol{y}):=-\frac{1}{2 \pi} \log |\boldsymbol{x}-\boldsymbol{y}|$ is the fundamental solution of the Laplacian in $\mathbb{R}^{2}, \lambda=\partial_{\nu} u$, and $c$ is a constant. Note that we made use of the transmission conditions (1.4). It is well known that (2.1) gives rise to the following system of boundary integral equations (see, e.g. 24]):

$$
\begin{aligned}
\mathcal{W} u-\left(\frac{1}{2} \mathcal{I}-\mathcal{K}^{\prime}\right) \lambda & =-\lambda \quad \text { on } \quad \Gamma, \\
\left(\frac{1}{2} \mathcal{I}-\mathcal{K}\right) u+\mathcal{V} \lambda+c & =0 \quad \text { on } \quad \Gamma,
\end{aligned}
$$

where $\mathcal{V}, \mathcal{K}, \mathcal{K}^{\prime}$, and $\mathcal{W}$ are the boundary integral operators associated with the single, double, adjoint of the double, and hypersingular layer potentials, respectively. We recall from 14 that $\mathcal{V}: H^{-1 / 2}(\Gamma) \rightarrow H^{1 / 2}(\Gamma), \mathcal{K}: H^{1 / 2}(\Gamma) \rightarrow H^{1 / 2}(\Gamma)$, $\mathcal{K}^{\prime}: H^{-1 / 2}(\Gamma) \rightarrow H^{-1 / 2}(\Gamma)$, and $\mathcal{W}: H^{1 / 2}(\Gamma) \rightarrow H^{-1 / 2}(\Gamma)$ are bounded linear operators, and that they are defined as follows:

$$
\begin{array}{rlrl}
\mathcal{V} \mu(\mathbf{x}) & :=\int_{\Gamma} E(\mathbf{x}, \boldsymbol{y}) \mu(\boldsymbol{y}) d s_{\boldsymbol{y}} & & \forall(\text { a.e. }) \mathbf{x} \in \Gamma, \forall \mu \in H^{-1 / 2}(\Gamma), \\
\mathcal{K} \psi(\mathbf{x}) & :=\int_{\Gamma} \partial_{\boldsymbol{\nu}(\boldsymbol{y})} E(\mathbf{x}, \boldsymbol{y}) \psi(\boldsymbol{y}) d s_{\boldsymbol{y}} & & (\text { a.e. }) \mathbf{x} \in \Gamma, \forall \psi \in H^{1 / 2}(\Gamma), \\
\mathcal{K}^{\prime} \mu(\mathbf{x}) & :=\int_{\Gamma} \partial_{\boldsymbol{\nu}(\mathbf{x})} E(\mathbf{x}, \boldsymbol{y}) \mu(\boldsymbol{y}) d s_{\boldsymbol{y}} & & \forall(\text { a.e. }) \mathbf{x} \in \Gamma, \forall \mu \in H^{-1 / 2}(\Gamma), \\
\mathcal{W} \psi(\mathbf{x}) & :=-\partial_{\boldsymbol{\nu}(\mathbf{x})} \int_{\Gamma} \partial_{\boldsymbol{\nu}(\boldsymbol{y})} E(\mathbf{x}, \boldsymbol{y}) \psi(\boldsymbol{y}) d s_{\boldsymbol{y}} & \forall(\text { a.e. }) \mathbf{x} \in \Gamma, \forall \psi \in H^{1 / 2}(\Gamma) .
\end{array}
$$

Here, $\partial_{\boldsymbol{\nu}(\mathbf{x})}$ stands for the normal derivative operator at $\mathbf{x} \in \Gamma$.

Next, according to the behavior of $u$ at infinity (cf. (1.1)), we observe that $\lambda$ belongs to $H_{0}^{-1 / 2}(\Gamma)$ where

$$
H_{0}^{-1 / 2}(\Gamma):=\left\{\mu \in H^{-1 / 2}(\Gamma): \quad\langle\mu, 1\rangle=0\right\} .
$$

According to the decomposition $H^{1 / 2}(\Gamma)=H_{0}^{1 / 2}(\Gamma) \oplus \mathbb{R}$, with

$$
H_{0}^{1 / 2}(\Gamma):=\left\{\psi \in H^{1 / 2}(\Gamma): \quad\langle 1, \psi\rangle=0\right\},
$$


we define

$$
\|\psi\|_{1 / 2, \Gamma, 0}:=\left\|\psi-\operatorname{length}(\Gamma)^{-1} \int_{\Gamma} \psi\right\|_{1 / 2, \Gamma} .
$$

Equivalently, $\|\cdot\|_{1 / 2, \Gamma, 0}$ denotes the quotient space norm

$$
\|\psi\|_{1 / 2, \Gamma, 0}:=\inf _{c \in \mathbb{R}}\|\psi+c\|_{1 / 2, \Gamma} \quad \forall \psi \in H^{1 / 2}(\Gamma) .
$$

The analysis of (2.2) and its discrete counterpart below will depend on the symmetry of $\mathcal{W}$ and the ellipticity of $\mathcal{V}$ and $\mathcal{W}$ :

$$
\begin{array}{rlrl}
\langle\mathcal{W} \varphi, \psi\rangle & =\langle\mathcal{W} \psi, \varphi\rangle & & \forall \varphi, \psi \in H^{1 / 2}(\Gamma), \\
\langle\mu, \mathcal{V} \mu\rangle & \geq C\|\mu\|_{-1 / 2, \Gamma}^{2} & \forall \mu \in H_{0}^{-1 / 2}(\Gamma), \\
\langle\mathcal{W} \psi, \psi\rangle & \geq C\|\psi\|_{1 / 2, \Gamma, 0}^{2} & \forall \psi \in H^{1 / 2}(\Gamma),
\end{array}
$$

2.2. The LDG formulation in the interior domain. The setting and analysis of the LDG formulation in $\Omega$ require several notations, definitions, and assumptions that we recall from [18. Let $\mathcal{T}_{h}$ be a shape regular triangulation of $\bar{\Omega}$ (with possible hanging nodes) made up of straight triangles $K$ with diameter $h_{K}$ and unit outward normal to $\partial K$ given by $\boldsymbol{\nu}_{K}$. As usual, the index $h$ denotes $h:=\max _{K \in \mathcal{T}_{h}} h_{K}$. Then, the edges of $\mathcal{T}_{h}$ are defined as follows. An interior edge of $\mathcal{T}_{h}$ is the (nonempty) interior of $\partial K \cap \partial K^{\prime}$ where $K$ and $K^{\prime}$ are two adjacent elements of $\mathcal{T}_{h}$. Similarly, a boundary edge of $\mathcal{T}_{h}$ is the (nonempty) interior of $\partial K \cap \Gamma_{0}$ or $\partial K \cap \Gamma$ where $K$ is an element of $\mathcal{T}_{h}$ which has an edge on $\Gamma_{0}$ or $\Gamma$. For each edge $e, h_{e}$ represents its length. In addition, we define $\mathcal{E}(K):=\{$ edges of $K\}, \mathcal{E}_{h}^{\text {int }}$ : set of interior edges (counted only once), $\mathcal{E}_{h}^{\Gamma}$ : set of edges on $\Gamma, \mathcal{E}_{h}^{\Gamma_{0}}$ : set of edges on $\Gamma_{0}$, and $I_{h}$ : interior grid generated by the triangulation, that is $I_{h}:=\bigcup\left\{e: e \in \mathcal{E}_{h}^{\text {int }}\right\}$. Also, we let $\Gamma_{h}$ and $\Gamma_{h}^{0}$ be the induced meshes on the boundaries $\Gamma$ and $\Gamma_{0}$, whose lists of edges are $\mathcal{E}_{h}^{\Gamma}$ and $\mathcal{E}_{h}^{\Gamma_{0}}$, respectively.

In what follows we assume that $\mathcal{T}_{h}$ is a locally quasi-uniform mesh, i.e., there exists $l>1$, independent of the meshsize $h$, such that $l^{-1} \leq \frac{h_{K}}{h_{K^{\prime}}} \leq l$ for each pair $K, K^{\prime} \in \mathcal{T}_{h}$ sharing an interior edge. We notice that the hypotheses on the triangulation imply that the cardinality of $\mathcal{E}(K)$ is uniformly bounded, and that

$$
h_{e} \leq h_{K} \leq C l h_{e}, \quad \forall e \in \mathcal{E}(K) .
$$

Now we consider integers $m \geq 1$ and $r \geq m-1 \geq 0$, and define the finite element spaces

$$
V_{h}:=\prod_{K \in \mathcal{T}_{h}} P_{m}(K) \quad \text { and } \quad \boldsymbol{\Sigma}_{h}:=\prod_{K \in \mathcal{T}_{h}} \mathbf{P}_{r}(K) .
$$

Hereafter, given an integer $k \geq 0$ and a domain $S \subseteq \mathbb{R}^{2}, P_{k}(S)$ denotes the space of polynomials of degree at most $k$ on $S$. For each $v:=\left\{v_{K}\right\}_{K \in \mathcal{T}_{h}} \in V_{h}$ and $\boldsymbol{\tau}:=$ $\left\{\boldsymbol{\tau}_{K}\right\}_{K \in \mathcal{T}_{h}} \in \boldsymbol{\Sigma}_{h}$, the components $v_{K}$ and $\boldsymbol{\tau}_{K}$ coincide with the restrictions $\left.v\right|_{K}$ and $\left.\boldsymbol{\tau}\right|_{K}$, when $v$ and $\boldsymbol{\tau}$ are identified as elements in $L^{2}(\Omega)$ and $\mathbf{L}^{2}(\Omega)$, respectively. Further, when no confusion arises, we omit the subscript $K$ and just write $v$ and $\tau$.

Next, consider the broken Sobolev spaces

$$
H^{s}\left(\mathcal{T}_{h}\right):=\prod_{K \in \mathcal{T}_{h}} H^{s}(K), \quad(s>1 / 2)
$$


as well as the spaces on the skeleton of the triangulation

$$
L^{2}\left(I_{h}\right):=\prod_{e \in \mathcal{E}_{h}^{\text {int }}} L^{2}(e), \quad P_{0}\left(I_{h}\right):=\prod_{e \in \mathcal{E}_{h}^{\text {int }}} P_{0}(e)
$$

and

$$
P_{0}\left(I_{h} \cup \Gamma_{h}^{0}\right):=\prod_{e \in \mathcal{E}_{h}^{\text {int }} \cup \mathcal{E}_{h}^{\Gamma_{0}}} P_{0}(e) .
$$

An analogue remark to the one given before, concerning components and restrictions of the elements in $V_{h}$ and $\boldsymbol{\Sigma}_{h}$, is valid here for each of the product spaces above. Also, we will not use any symbol for the trace on edges, provided it is clear from which side of an interior edge we are taking the trace. Hence, given $v \in H^{1}\left(\mathcal{T}_{h}\right)$, we define the averages $\{v\} \in L^{2}\left(I_{h}\right)$ and jumps $\llbracket v \rrbracket \in \mathbf{L}^{2}\left(I_{h}\right)$ on the interior grid $I_{h}$ by

$$
\{v\}_{e}:=\frac{1}{2}\left(v_{K}+v_{K^{\prime}}\right) \quad \text { and } \quad \llbracket v \rrbracket_{e}:=v_{K} \boldsymbol{\nu}_{K}+v_{K^{\prime}} \boldsymbol{\nu}_{K^{\prime}} \quad \forall e \in \mathcal{E}(K) \cap \mathcal{E}\left(K^{\prime}\right) .
$$

Similarly, for vector-valued functions $\boldsymbol{\tau} \in \mathbf{H}^{1}\left(\mathcal{T}_{h}\right)$, we define $\{\boldsymbol{\tau}\} \in \mathbf{L}^{2}\left(I_{h}\right)$ and $\llbracket \tau \rrbracket \in L^{2}\left(I_{h}\right)$ by

$$
\{\boldsymbol{\tau}\}_{e}:=\frac{1}{2}\left(\boldsymbol{\tau}_{K}+\boldsymbol{\tau}_{K^{\prime}}\right) \quad \text { and } \quad \llbracket \boldsymbol{\tau} \rrbracket_{e}:=\boldsymbol{\tau}_{K} \cdot \boldsymbol{\nu}_{K}+\boldsymbol{\tau}_{K^{\prime}} \cdot \boldsymbol{\nu}_{K^{\prime}} \quad \forall e \in \mathcal{E}(K) \cap \mathcal{E}\left(K^{\prime}\right) .
$$

In addition, let $\alpha \in P_{0}\left(I_{h} \cup \Gamma_{h}^{0}\right)$ and $\boldsymbol{\beta} \in \mathbf{P}_{0}\left(I_{h}\right)$ be given functions and assume that there exist $C, c_{0}, c_{1}>0$, independent of the grid, such that

$$
\max _{e \in \mathcal{E}_{h}^{\text {int }}}\left|\boldsymbol{\beta}_{e}\right| \leq C \quad \text { and } \quad 0<c_{0} \leq h_{\mathcal{E}} \alpha \leq c_{1}
$$

where $h_{\mathcal{E}} \in P_{0}\left(I_{h} \cup \Gamma_{h}^{0}\right)$ is defined by $\left.h_{\mathcal{E}}\right|_{e}:=h_{e} \quad \forall e \in \mathcal{E}_{h}^{\text {int }} \cup \mathcal{E}_{h}^{\Gamma_{0}}$.

We are now in a position to introduce the LDG scheme for the interior problem (1.2). As usual, we first define the gradient $\sigma:=\nabla u$ in $\Omega$ as an additional unknown where $u$ is the exact solution of (1.2)-(1.3). Then, let $\lambda_{h} \in L^{2}(\Gamma)$ be a discrete approximation (to be defined below) of the normal derivative $\lambda$, and proceeding as in [12, 18, we arrive at the following global LDG formulation: Find $\left(\boldsymbol{\sigma}_{h}, u_{h}\right) \in \boldsymbol{\Sigma}_{h} \times V_{h}$ such that

$$
\begin{array}{ll}
\int_{\Omega} \boldsymbol{\sigma}_{h} \cdot \boldsymbol{\tau}-\left\{\int_{\Omega} \nabla_{h} u_{h} \cdot \boldsymbol{\tau}-S\left(u_{h}, \boldsymbol{\tau}\right)\right\}=0 & \forall \boldsymbol{\tau} \in \boldsymbol{\Sigma}_{h}, \\
\left\{\int_{\Omega} \nabla_{h} v \cdot \boldsymbol{\sigma}_{h}-S\left(v, \boldsymbol{\sigma}_{h}\right)\right\}+\boldsymbol{\alpha}\left(u_{h}, v\right)=\int_{\Omega} f v+\int_{\Gamma} \lambda_{h} v & \forall v \in V_{h},
\end{array}
$$

where $\nabla_{h}$ stands for the piecewise defined gradient, and $S: H^{1}\left(\mathcal{T}_{h}\right) \times \mathbf{H}^{1}\left(\mathcal{T}_{h}\right) \rightarrow \mathbb{R}$ and $\boldsymbol{\alpha}: H^{1}\left(\mathcal{T}_{h}\right) \times H^{1}\left(\mathcal{T}_{h}\right) \rightarrow \mathbb{R}$ are the bilinear forms defined by

$S(w, \boldsymbol{\tau}):=\int_{I_{h}} \llbracket w \rrbracket \cdot(\{\boldsymbol{\tau}\}-\llbracket \boldsymbol{\tau} \rrbracket \boldsymbol{\beta})+\int_{\Gamma_{0}} w(\boldsymbol{\tau} \cdot \boldsymbol{\nu}) \quad \forall(w, \boldsymbol{\tau}) \in H^{1}\left(\mathcal{T}_{h}\right) \times \mathbf{H}^{1}\left(\mathcal{T}_{h}\right)$,

and

$$
\boldsymbol{\alpha}(w, v):=\int_{I_{h}} \alpha \llbracket w \rrbracket \cdot \llbracket v \rrbracket+\int_{\Gamma_{0}} \alpha w v \quad \forall(w, v) \in H^{1}\left(\mathcal{T}_{h}\right) \times H^{1}\left(\mathcal{T}_{h}\right),
$$

with the traces of $w, v$, and $\tau$ on $\Gamma_{0}$ being defined elementwise. 
2.3. The coupled LDG-BEM scheme. We now establish the coupled LDGBEM scheme by combining a discrete form of (2.2) with the LDG formulation (2.8). This requires a subspace for $\lambda_{h}$ and an approximant $u_{h}$ of $u$ which is continuous on $\Gamma$. For the discrete space approximating $\lambda$ we take, for simplicity, the partition of $\Gamma$ induced by $\mathcal{T}_{h}$ and introduce

$$
X_{h}:=\prod_{e \in \mathcal{E}_{h}^{\Gamma}} P_{m-1}(e) \quad \text { and } \quad X_{h}^{0}:=\left\{\mu_{h} \in X_{h}: \int_{\Gamma} \mu_{h}=0\right\}=X_{h} \cap H_{0}^{-1 / 2}(\Gamma) .
$$

Then, we consider the subspace $\tilde{V}_{h}$ of $V_{h}$ defined by

$$
\tilde{V}_{h}:=\left\{v_{h} \in V_{h}:\left.\quad v_{h}\right|_{\Gamma} \in C(\Gamma)\right\}
$$

Here, the trace $\left.v_{h}\right|_{\Gamma}$ for $v_{h} \in V_{h}$ is defined in a piecewise manner on the edges of $\Gamma_{h}$ and the condition $\left.v_{h}\right|_{\Gamma} \in C(\Gamma)$ means that the function composed by the piecewise traces is continuous on $\Gamma$. Hence, substituting $\lambda_{h}$ in (2.8) by a discrete version of the first equation in (2.2), in which $u$ is replaced by its approximant $u_{h}$, and adding also a discrete formulation of the second equation in (2.2), we obtain the following coupled LDG-BEM scheme: Find $\left(\boldsymbol{\sigma}_{h}, u_{h}, \lambda_{h}\right) \in \boldsymbol{\Sigma}_{h} \times \tilde{V}_{h} \times X_{h}^{0}$ such that

$$
\begin{aligned}
\int_{\Omega} \boldsymbol{\sigma}_{h} \cdot \boldsymbol{\tau}-\boldsymbol{\rho}\left(u_{h}, \boldsymbol{\tau}\right) & =0 \\
\boldsymbol{\rho}\left(v, \boldsymbol{\sigma}_{h}\right)+\boldsymbol{\alpha}\left(u_{h}, v\right)+\left\langle\mathcal{W} u_{h}, v\right\rangle-\left\langle\left(\frac{1}{2} \mathcal{I}-\mathcal{K}^{\prime}\right) \lambda_{h}, v\right\rangle & =\int_{\Omega} f v, \\
\left\langle\mu,\left(\frac{1}{2} \mathcal{I}-\mathcal{K}\right) u_{h}\right\rangle+\left\langle\mu, \mathcal{V} \lambda_{h}\right\rangle & =0
\end{aligned}
$$

for all $(\boldsymbol{\tau}, v, \mu) \in \boldsymbol{\Sigma}_{h} \times \tilde{V}_{h} \times X_{h}^{0}$, where $\boldsymbol{\rho}: H^{1}\left(\mathcal{T}_{h}\right) \times \mathbf{H}^{1}\left(\mathcal{T}_{h}\right) \rightarrow \mathbb{R}$ is the bilinear form defined by

$$
\boldsymbol{\rho}(v, \boldsymbol{\tau}):=\int_{\Omega} \nabla_{h} v \cdot \boldsymbol{\tau}-S(v, \boldsymbol{\tau}) \quad \forall(v, \boldsymbol{\tau}) \in H^{1}\left(\mathcal{T}_{h}\right) \times \mathbf{H}^{1}\left(\mathcal{T}_{h}\right) .
$$

This coupled LDG-BEM scheme has the usual form of the traditional coupling of finite and boundary elements (see [13, 20]): diagonal operators are symmetric and off-diagonal operators form a skew symmetric matrix. The complete system can be made symmetric (although indefinite) by changing the sign of the second equation. Also, notice that occurrences of $u_{h}$ as well as $v \in \tilde{V}_{h}$ inside the duality bracket and under the action of integral operators include the use of the piecewise trace which belongs to $H^{1 / 2}(\Gamma)$.

In order to compare the formulation (2.12) with the one from 18 we recall that the latter is given as follows: Find $\left(\boldsymbol{\sigma}_{h}, u_{h}, \lambda_{\tilde{h}}, \varphi_{\hat{h}}, \gamma_{\hat{h}}\right) \in \Sigma_{h} \times V_{h} \times X_{\tilde{h}}^{0} \times Y_{\hat{h}}^{0} \times Z_{\hat{h}}^{0}$ 
such that

$$
\begin{aligned}
\int_{\Omega} \boldsymbol{\sigma}_{h} \cdot \boldsymbol{\tau}-\boldsymbol{\rho}\left(u_{h}, \boldsymbol{\tau}\right) & =0, \\
\boldsymbol{\rho}\left(v, \boldsymbol{\sigma}_{h}\right)+\boldsymbol{\alpha}\left(u_{h}, v\right)-\left\langle\lambda_{\tilde{h}}, v\right\rangle & =\int_{\Omega} f v, \\
\left\langle\xi, u_{h}\right\rangle-\left\langle\xi, \varphi_{\hat{h}}\right\rangle & =0, \\
\left\langle\lambda_{\hat{h}}, \psi\right\rangle+\left\langle\mathcal{W} \varphi_{\hat{h}}, \psi\right\rangle-\left\langle\left(\frac{1}{2} \mathcal{I}-\mathcal{K}^{\prime}\right) \gamma_{\hat{h}}, \psi\right\rangle & =0, \\
\left\langle\mu,\left(\frac{1}{2} \mathcal{I}-\mathcal{K}\right) \varphi_{\hat{h}}\right\rangle+\left\langle\mu, \mathcal{V} \gamma_{\hat{h}}\right\rangle & =0
\end{aligned}
$$

for all $(\boldsymbol{\tau}, v, \xi, \psi, \mu) \in \boldsymbol{\Sigma}_{h} \times V_{h} \times X_{\tilde{h}}^{0} \times Y_{\hat{h}}^{0} \times Z_{\hat{h}}^{0}$, where $X_{\tilde{h}}^{0} \subseteq L^{2}(\Gamma) \cap H_{0}^{-1 / 2}(\Gamma), Y_{\hat{h}}^{0} \subseteq$ $C(\Gamma) \cap H_{0}^{1 / 2}(\Gamma)$, and $Z_{\hat{h}}^{0} \subseteq L^{2}(\Gamma) \cap H_{0}^{-1 / 2}(\Gamma)$ are boundary element subspaces, with independent meshsizes $\tilde{h}$ and $\hat{h}$, for the mortar-type auxiliary unknown $\lambda_{\tilde{h}}$ gluing the LDG and BEM modules, and for the Cauchy data $\varphi_{\hat{h}}$ and $\gamma_{\hat{h}}$, respectively. We observe that the computational implementation of (2.14) can be easily obtained by incorporating individual codes for each module, which constitutes the main advantage of this formulation, whereas the lower number of unknowns involved is the main strength of the present approach (2.12).

Now, for the solvability and stability of (2.12) we need an equivalent reduced formulation which is taken from [18]. To this end let $\mathbf{S}_{h}: H^{1}\left(\mathcal{T}_{h}\right) \rightarrow \boldsymbol{\Sigma}_{h}$ be the linear operator associated with the bilinear form $S$ restricted to $H^{1}\left(\mathcal{T}_{h}\right) \times \boldsymbol{\Sigma}_{h}$. That is, given $w \in H^{1}\left(\mathcal{T}_{h}\right), \mathbf{S}_{h}(w)$ is the unique element in $\boldsymbol{\Sigma}_{h}$ satisfying

$$
\int_{\Omega} \mathbf{S}_{h}(w) \cdot \boldsymbol{\tau}=S(w, \boldsymbol{\tau}) \quad \forall \boldsymbol{\tau} \in \mathbf{\Sigma}_{h} .
$$

Next, let $B_{h}: H^{1}\left(\mathcal{T}_{h}\right) \times H^{1}\left(\mathcal{T}_{h}\right) \rightarrow \mathbb{R}$ be the bilinear form defined by

$$
B_{h}(w, v):=\boldsymbol{\alpha}(w, v)+\int_{\Omega}\left(\nabla_{h} w-\mathbf{S}_{h}(w)\right) \cdot\left(\nabla_{h} v-\mathbf{S}_{h}(v)\right) \quad \forall w, v \in H^{1}\left(\mathcal{T}_{h}\right) .
$$

The equivalence between (2.12) and a reduced problem involving $B_{h}$ is established by the following lemma.

Lemma 2.1. Let $\left(\boldsymbol{\sigma}_{h}, u_{h}, \lambda_{h}\right) \in \boldsymbol{\Sigma}_{h} \times \tilde{V}_{h} \times X_{h}^{0}$ be a solution of (2.12). Then it holds that

$$
\begin{aligned}
B_{h}\left(u_{h}, v\right)+\left\langle\mathcal{W} u_{h}, v\right\rangle-\left\langle\left(\frac{1}{2} \mathcal{I}-\mathcal{K}^{\prime}\right) \lambda_{h}, v\right\rangle & =\int_{\Omega} f v, \\
\left\langle\mu,\left(\frac{1}{2} \mathcal{I}-\mathcal{K}\right) u_{h}\right\rangle+\left\langle\mu, \mathcal{V} \lambda_{h}\right\rangle & =0
\end{aligned}
$$

for any $(v, \mu) \in \tilde{V}_{h} \times X_{h}^{0}$. Conversely, if $\left(u_{h}, \lambda_{h}\right) \in \tilde{V}_{h} \times X_{h}^{0}$ satisfies (2.17) and $\boldsymbol{\sigma}_{h}:=\nabla_{h} u_{h}-\mathbf{S}_{h}\left(u_{h}\right)$, then $\left(\boldsymbol{\sigma}_{h}, u_{h}, \lambda_{h}\right)$ is a solution of (2.12). If $\left(u_{h}, \lambda_{h}\right) \in$ $\tilde{V}_{h} \times X_{h}^{0}$ is the only solution of (2.17), then $\left(\boldsymbol{\sigma}_{h}, u_{h}, \lambda_{h}\right)$, with $\boldsymbol{\sigma}_{h}$ defined as before, is the only solution of (2.12).

Proof. This result is analogous to Lemma 2.2 in [18 and is based on the fact that the first equation in (2.12) can be written as

$$
\int_{\Omega} \boldsymbol{\sigma}_{h} \cdot \boldsymbol{\tau}-\int_{\Omega}\left(\nabla_{h} u_{h}-\mathbf{S}_{h}\left(u_{h}\right)\right) \cdot \boldsymbol{\tau}=0 \quad \forall \boldsymbol{\tau} \in \boldsymbol{\Sigma}_{h} .
$$


The fact that $r \geq m-1$ guarantees that $\nabla_{h} u_{h} \in \boldsymbol{\Sigma}_{h}$, which yields $\boldsymbol{\sigma}_{h}=\nabla_{h} u_{h}-$ $\mathbf{S}_{h}\left(u_{h}\right)$ and leads to the result.

\section{UNIQUE SOLVABILITY AND STABILITY}

In this section we prove the unique solvability and stability of (2.12) through the corresponding analysis of the equivalent reduced formulation (2.17). We first introduce seminorms

$$
|v|_{1, h}^{2}:=\left\|\nabla_{h} v\right\|_{0, \Omega}^{2}, \quad|v|_{*}^{2}:=\left\|h_{\mathcal{E}}^{-1 / 2} \llbracket v \rrbracket\right\|_{0, I_{h}}^{2}+\left\|h_{\mathcal{E}}^{-1 / 2} v\right\|_{0, \Gamma_{0}}^{2} \quad \forall v \in H^{1}\left(\mathcal{T}_{h}\right),
$$

and the norm

$$
\|v\|_{h}^{2}:=|v|_{1, h}^{2}+|v|_{*}^{2} \quad \forall v \in H^{1}\left(\mathcal{T}_{h}\right) .
$$

Next, we let $\mathbf{B}_{h}$ denote the bilinear form defined by the left-hand side of (2.17), i.e.

$$
\begin{aligned}
\mathbf{B}_{h}(w, \eta ; v, \mu):= & B_{h}(w, v)+\langle\mathcal{W} w, v\rangle \\
& -\left\langle\left(\frac{1}{2} \mathcal{I}-\mathcal{K}^{\prime}\right) \eta, v\right\rangle+\left\langle\mu,\left(\frac{1}{2} \mathcal{I}-\mathcal{K}\right) w\right\rangle+\langle\mu, \mathcal{V} \eta\rangle
\end{aligned}
$$

for

$$
w, v \in H_{1 / 2}^{1}\left(\mathcal{T}_{h}\right):=\left\{w \in H^{1}\left(\mathcal{T}_{h}\right):\left.\quad w\right|_{\Gamma} \in H^{1 / 2}(\Gamma)\right\}
$$

and $\eta, \mu \in H_{0}^{-1 / 2}(\Gamma)$. Analogously as before, the trace $\left.w\right|_{\Gamma} \in L^{2}(\Gamma)$ for $w \in H^{1}\left(\mathcal{T}_{h}\right)$ is defined first on each edge of $\Gamma_{h}$ and the condition $\left.w\right|_{\Gamma} \in H^{1 / 2}(\Gamma)$ means that the function composed by the piecewise traces is in $H^{1 / 2}(\Gamma)$.

The full discrete norm, defined for elements $(v, \mu) \in H_{1 / 2}^{1}\left(\mathcal{T}_{h}\right) \times H_{0}^{-1 / 2}(\Gamma)$ will be given by the expression

$$
\|(v, \mu)\|_{h, \Gamma}^{2}:=\|v\|_{h}^{2}+\|v\|_{1 / 2, \Gamma, 0}^{2}+\|\mu\|_{-1 / 2, \Gamma}^{2} .
$$

Essential ingredients of our analysis are the properties of the bilinear form $\mathbf{B}_{h}$ with respect to this norm.

Lemma 3.1. There exist positive constants $c, C$, independent of $h$, such that

$$
\left|\mathbf{B}_{h}(w, \eta ; v, \mu)\right| \leq c\|(w, \eta)\|_{h, \Gamma}\|(v, \mu)\|_{h, \Gamma} \quad \forall(w, \eta),(v, \mu) \in H_{1 / 2}^{1}\left(\mathcal{T}_{h}\right) \times H_{0}^{-1 / 2}(\Gamma)
$$

and

$$
\mathbf{B}_{h}(v, \mu ; v, \mu) \geq C\|(v, \mu)\|_{h, \Gamma}^{2} \quad \forall(v, \mu) \in H_{1 / 2}^{1}\left(\mathcal{T}_{h}\right) \times H_{0}^{-1 / 2}(\Gamma) .
$$

Proof. Recall first that by [18, Lemma 3.2], there exist positive constants $c, C$, independent of $h$, such that

$$
\left|B_{h}(w, v)\right| \leq c\|w\|_{h}\|v\|_{h} \quad \forall w, v \in H^{1}\left(\mathcal{T}_{h}\right)
$$

and

$$
B_{h}(v, v) \geq C\|v\|_{h}^{2} \quad \forall v \in H^{1}\left(\mathcal{T}_{h}\right) .
$$

According to the properties of the operators $\mathcal{V}, \mathcal{W}$ and $\mathcal{K}$ (cf. Section 2.1), noting that $\mathcal{W} 1=0$ and $\mathcal{K} 1=-\frac{1}{2}$ on $\Gamma$, and using the decomposition $H^{1 / 2}(\Gamma)=$ $H_{0}^{1 / 2}(\Gamma) \oplus \mathbb{R}$ and the definition of the seminorm $\|\cdot\|_{1 / 2, \Gamma, 0}(\mathrm{cf}$. (2.3) $)$, we find that

$$
\begin{aligned}
& |\langle\mu, \mathcal{V} \eta\rangle| \leq C\|\mu\|_{-1 / 2, \Gamma}\|\eta\|_{-1 / 2, \Gamma} \quad \forall \mu, \eta \in H_{0}^{-1 / 2}(\Gamma), \\
& |\langle\mathcal{W} w, v\rangle| \leq C\|w\|_{1 / 2, \Gamma, 0}\|v\|_{1 / 2, \Gamma, 0} \quad \forall w, v \in H_{1 / 2}^{1}\left(\mathcal{T}_{h}\right),
\end{aligned}
$$


and

$$
\left|\left\langle\mu,\left(\frac{1}{2} \mathcal{I}-\mathcal{K}\right) w\right\rangle\right| \leq C\|w\|_{1 / 2, \Gamma, 0}\|\mu\|_{-1 / 2, \Gamma} \quad \forall(w, \mu) \in H_{1 / 2}^{1}\left(\mathcal{T}_{h}\right) \times H_{0}^{-1 / 2}(\Gamma) .
$$

The inequalities above and (3.4) yield the continuity estimate (3.2) for $\mathbf{B}_{h}$. Next, we observe from the definition of $\mathbf{B}_{h}$ that

$$
\mathbf{B}_{h}(v, \mu ; v, \mu)=B_{h}(v, v)+\langle\mathcal{W} v, v\rangle+\langle\mu, \mathcal{V} \mu\rangle \quad \forall(v, \mu) \in H_{1 / 2}^{1}\left(\mathcal{T}_{h}\right) \times H_{0}^{-1 / 2}(\Gamma),
$$

and hence, (2.4) and (3.5) imply the ellipticity estimate (3.3) for $\mathbf{B}_{h}$.

We are now in a position to prove the unique solvability and stability of (2.12).

Theorem 3.1. The coupled LDG-BEM scheme (2.12) is uniquely solvable and the stability estimate below holds:

$$
\left\|\boldsymbol{\sigma}_{h}\right\|_{0, \Omega}+\left\|\left(u_{h}, \lambda_{h}\right)\right\|_{h, \Gamma} \leq C\|f\|_{0, \Omega} .
$$

Proof. By Lemma2.1 it suffices to study the system (2.17) instead of (2.12). Indeed, the ellipticity of $\mathbf{B}_{h}$ (cf. Lemma 3.1) implies the unique solvability of (2.17), and using additionally that $\|v\|_{0, \Omega} \leq C\|v\|_{h} \forall v \in V_{h}$ (see [1]), we deduce the stability estimate

$$
\left\|\left(u_{h}, \lambda_{h}\right)\right\|_{h, \Gamma} \leq C\|f\|_{0, \Omega} .
$$

By Lemma 2.1 we then conclude the unique solvability of (2.12). By equation (3.11) in [18] it holds that

$$
\left\|\mathbf{S}_{h}(w)\right\|_{0, \Omega} \leq C|w|_{*} \quad \forall w \in H^{1}\left(\mathcal{T}_{h}\right) .
$$

Therefore, making use of the relation $\boldsymbol{\sigma}_{h}=\nabla_{h} u_{h}-\mathbf{S}_{h}\left(u_{h}\right)$, we find that

$$
\left\|\boldsymbol{\sigma}_{h}\right\|_{0, \Omega} \leq C\left\|u_{h}\right\|_{h} \leq C\|f\|_{0, \Omega},
$$

which finishes the proof of the theorem.

\section{A PRIORI ERROR ANALYSIS}

In order to derive the a priori error estimate of the coupled scheme some technical results are needed. Because of (2.5) it is easy to see that

$$
\|u\|_{h}^{2} \leq C \sum_{K \in \mathcal{T}_{h}}\left(\|\nabla u\|_{0, K}^{2}+\left\|h_{K}^{-1 / 2} u\right\|_{0, \partial K}^{2}\right) \quad \forall u \in H^{1}\left(\mathcal{T}_{h}\right) .
$$

In what follows let $\hat{K}$ denote the reference triangle

$$
\hat{K}:=\left\{\left(x_{1}, x_{2}\right): 0<x_{1}<1,0<x_{2}<1-x_{1}\right\} .
$$

For any $K \in \mathcal{T}_{h}$ we choose an invertible affine map $M_{K}: \hat{K} \rightarrow K$. As usual in the finite element literature, given $u: K \rightarrow \mathbb{R}$ we will denote $\hat{u}:=u \circ M_{K}: \hat{K} \rightarrow \mathbb{R}$.

We begin by recalling some local approximation properties. The following result rephrases [6, Lemma 4.1], which itself collects several results from [3, 4]. 
Lemma 4.1. Given an integer $m \geq 1$, there exists an operator $\hat{\boldsymbol{\pi}}: L^{2}(\hat{K}) \rightarrow$ $P_{m}(\hat{K})$ such that for all $u \in H^{k}(K)$,

$$
\begin{gathered}
\|\hat{u}-\hat{\boldsymbol{\pi}} \hat{u}\|_{q, \hat{K}} \leq \frac{C}{m^{k-q}} h^{\mu}\|u\|_{k, K}, 0 \leq q \leq k, \\
\sup _{\hat{x} \in \hat{K}}|(\hat{u}-\hat{\boldsymbol{\pi}} \hat{u})(\hat{x})| \leq \frac{C}{m^{k-1}} h^{\mu}\|u\|_{k, K}, \quad k>1, \\
\|\hat{u}-\hat{\boldsymbol{\pi}} \hat{u}\|_{s, \partial \hat{K}} \leq \frac{C}{m^{k-s-1 / 2}} h^{\mu}\|u\|_{k, K}, \quad k>3 / 2, \quad s \in\{0,1\},
\end{gathered}
$$

where $\mu=\min \{k-1, m\}$. The constant $C$ depends only on $k$.

The following lemma, whose proof below makes extensive use of the estimates (4.2) -(4.4), provides a global approximation property of the subspace $\tilde{V}_{h}$.

Lemma 4.2. Assume that $u \in H^{1+\delta}(\Omega)$ for some $\delta>1 / 2$. Then there exists $v_{h} \in \tilde{V}_{h}$ such that

$$
\left\|u-v_{h}\right\|_{h}+\left\|u-v_{h}\right\|_{1 / 2, \Gamma, 0} \leq C h^{\min \{\delta, m\}}\|u\|_{1+\delta, \Omega} .
$$

Here, $C>0$ is a constant independent of $h$.

Proof. Let $\bar{v}_{h} \in V_{h}$ be constructed using locally the operator of Lemma4.1 Namely, let $u_{K}:=\left.u\right|_{K}$ for each $K \in \mathcal{T}_{h}$ and, as usual, $\hat{u}_{K}:=u_{K} \circ M_{K}$. Then we define $\left.\bar{v}_{h}\right|_{K}:=\left(\hat{\boldsymbol{\pi}} \hat{u}_{K}\right) \circ M_{K}^{-1}$. Taking into account the scaling properties of the norms and applying (4.2) and (4.4) we obtain

$$
\begin{aligned}
\left|u-\bar{v}_{h}\right|_{1, K}^{2}+\left\|h_{K}^{-1 / 2}\left(u-\bar{v}_{h}\right)\right\|_{0, \partial K}^{2} & \leq C\left(\left|\hat{u}_{K}-\hat{\pi} \hat{u}_{K}\right|_{1, K}^{2}+\left\|\hat{u}_{K}-\hat{\pi} \hat{u}_{K}\right\|_{0, \partial \hat{K}}^{2}\right) \\
& \leq C^{\prime} h^{2 \min \{\delta, m\}}\|u\|_{1+\delta, K}^{2},
\end{aligned}
$$

since $\delta>1 / 2$. Adding the contributions of the different triangles and using (4.1) we have proved that

$$
\left\|u-\bar{v}_{h}\right\|_{h} \leq C h^{\min \{\delta, m\}}\|u\|_{1+\delta, \Omega} .
$$

We now correct the value of $\bar{v}_{h}$ only on triangles with an edge on $\Gamma$, in such a way that we construct $v_{h} \in \tilde{V}_{h}$ with the same order of approximation as $\bar{v}_{h}$.

The technique is standard in finite element analysis. Let $\hat{P}_{1}:=(0,0), \hat{P}_{2}:=$ $(1,0)$ and $\hat{P}_{3}:=(0,1)$ be the three vertices of $\hat{K}$. Consider also the functions $\hat{N}_{1}\left(x_{1}, x_{2}\right):=1-x_{1}-x_{2}$ and $\hat{N}_{2}\left(x_{1}, x_{2}\right):=x_{2}$. Consider the map $\hat{C}: \mathcal{C}(\hat{K}) \rightarrow P_{1}(\hat{K})$ given by

$$
\hat{C} \hat{u}:=\hat{u}\left(\hat{P}_{1}\right) \hat{N}_{1}+\hat{u}\left(\hat{P}_{2}\right) \hat{N}_{2}
$$

which yields a linear interpolant of $\hat{u}$ on the edge connecting $\hat{P}_{1}$ and $\hat{P}_{2}$ (ê in Figure 1) and makes $(\hat{C} \hat{u})\left(\hat{P}_{3}\right)=0$. We then correct $\hat{\boldsymbol{\pi}}$ in the following form:

$$
\hat{\Pi} \hat{u}:=\hat{\boldsymbol{\pi}} \hat{u}+\hat{C}(\hat{u}-\hat{\boldsymbol{\pi}} \hat{u})=\hat{\boldsymbol{\pi}} \hat{u}-\hat{C}(\hat{\boldsymbol{\pi}} \hat{u})+\hat{C} \hat{u}, \quad \hat{u} \in \mathcal{C}(\hat{K}) .
$$

Notice that $(\hat{\Pi} \hat{u})\left(\hat{P}_{j}\right)=\hat{u}\left(\hat{P}_{j}\right)$ for $j=1$ and 2 , whereas $(\hat{\Pi} \hat{u})\left(\hat{P}_{3}\right)=(\hat{\boldsymbol{\pi}} \hat{u})\left(\hat{P}_{3}\right)$. Using (4.2), (4.3) and (4.4) we can easily prove that if $u \in H^{1+\delta}(K)$, with $\delta>1 / 2$, then

$$
\begin{aligned}
|\hat{u}-\hat{\Pi} \hat{u}|_{1, \hat{K}}+\|\hat{u}-\hat{\Pi} u\|_{0, \partial \hat{K}} \leq|\hat{u}-\hat{\boldsymbol{\pi}} u|_{1, \hat{K}}+\|\hat{u}-\hat{\boldsymbol{\pi}} \hat{u}\|_{0, \partial \hat{K}} & \\
& +C \max _{j=1,2}\left|\hat{u}\left(\hat{P}_{j}\right)-(\hat{\boldsymbol{\pi}} \hat{u})\left(\hat{P}_{j}\right)\right| \\
\leq & C h^{\min \{\delta, m\}}\|u\|_{1+\delta, K} .
\end{aligned}
$$



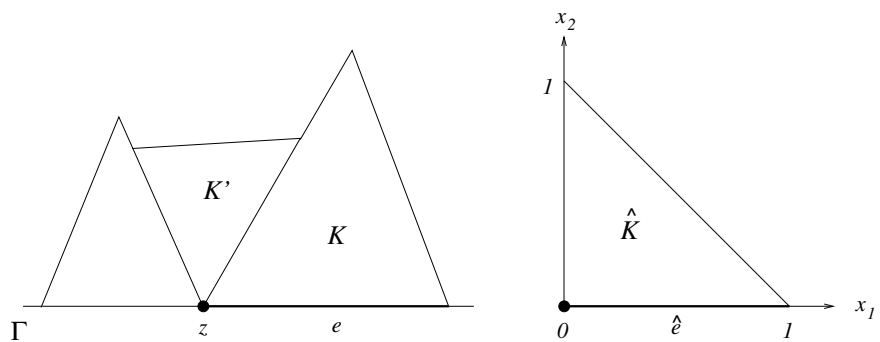

Figure 1. Adjusting to continuity at the boundary. The value on $z$ of the approximation is changed for $K$ but not for $K^{\prime}$.

We then construct $v_{h}$ as follows. If $K$ does not have an edge on $\Gamma$, we take $\left.v_{h}\right|_{K}:=$ $\left.\bar{v}_{h}\right|_{K}$. If the edge $e \in \mathcal{E}(K)$ is contained in $\Gamma$, we take the map $M_{K}: \hat{K} \rightarrow K$ so that the side $\hat{e}:=[0,1] \times\{0\}$ is mapped onto $e$. Then $\left.v_{h}\right|_{K}:=\left(\hat{\Pi} \hat{u}_{K}\right) \circ M_{K}^{-1}$. Notice that if $\boldsymbol{z}$ is one vertex of $e$, then $v_{h}(\boldsymbol{z})=u(\boldsymbol{z})$. Therefore the restriction of $v_{h}$ to the boundary is continuous (see Figure 11). The same arguments as we used for $\bar{v}_{h}$ together with (4.7) prove that

$$
\left\|u-v_{h}\right\|_{h} \leq C h^{\min \{\delta, m\}}\|u\|_{1+\delta, \Omega} .
$$

In order to conclude (4.5) it just remains to show that

$$
\left\|u-v_{h}\right\|_{1 / 2, \Gamma, 0} \leq C h^{\min \{\delta, m\}}\|u\|_{1+\delta, \Omega} .
$$

Notice that by interpolation of norms

$$
\begin{aligned}
\| u- & v_{h}\left\|_{1 / 2, \Gamma, 0} \leq\right\| u-v_{h} \|_{1 / 2, \Gamma} \\
\leq & \leq\left(\sum_{e \in \mathcal{E}_{h}^{\Gamma}}\left\|u-v_{h}\right\|_{0, e}^{2}\right)^{1 / 2}\left(\sum_{e \in \mathcal{E}_{h}^{\Gamma}}\left\|u-v_{h}\right\|_{1, e}^{2}\right)^{1 / 2} .
\end{aligned}
$$

Moving to the boundary of the reference domain and again using (4.3) and (4.4) we easily prove that for $s \in\{0,1\}$,

$$
\left\|u-v_{h}\right\|_{s, \partial K} \leq C h_{K}^{1 / 2-s}\left\|\hat{u}_{K}-\hat{\Pi} \hat{u}_{K}\right\|_{s, \partial \hat{K}} \leq C h^{\min \{\delta, m\}+1 / 2-s}\|u\|_{1+\delta, K} .
$$

Since all the terms in the right-hand side of (4.10) can be bounded by the estimate above (take the triangle $K$ such that $e \in \mathcal{E}(K)$ ), then (4.9) follows readily.

We note that defining $\bar{v}_{h}$ as the $L^{2}(\Omega)$-orthogonal projection of $u$ onto $V_{h}$ would also yield the estimate (4.6) (see Lemmas 4.2 and 4.4 in [18 for details). However, this choice of $\bar{v}_{h}$ does not allow the further construction of $v_{h} \in \tilde{V}_{h}$ satisfying the approximation property (4.5). This is the reason why we proceed differently and employ the local approximant provided by Lemma 4.1 .

Next, we derive an approximation property for the subspace $X_{h}^{0}$. First let us clarify some notation. For $|t| \leq 1$ the spaces $H^{t}(\Gamma)$ are well defined in a classical way. Let $\left\{\Gamma_{1}, \ldots, \Gamma_{N}\right\}$ denote the edges of the polygon $\Gamma$. For $t \geq 0$, we define $H^{t}\left(\Gamma_{j}\right)$ as the space of functions that can be extended to a function in $H^{t}(\mathbb{R})$, after identification of $\Gamma_{j}$ with an interval on the real line. This space is endowed with the image topology of the restriction operator. Finally, we denote $H_{\text {prod }}^{t}(\Gamma):=$ $\prod_{j} H^{t}\left(\Gamma_{j}\right)$ and denote its norm by $\|\cdot\|_{t, \operatorname{prod}, \Gamma}$. Since the normal vector field is 
constant on each edge, it is easy to see that if $u \in H^{1+\delta}(\Omega)$, with $\delta>1 / 2$, then $\lambda:=\partial_{\nu} u \in H_{\text {prod }}^{\delta-1 / 2}(\Gamma)$ and

$$
\|\lambda\|_{\delta-1 / 2, \operatorname{prod}, \Gamma} \leq C\|u\|_{1+\delta, \Omega} .
$$

For the particular form of the precise image space of the trace and normal derivative operators on polygons see [19]. Note that for $0<t \leq 1, H^{t}(\Gamma)$ is a closed subspace of $H_{\text {prod }}^{t}(\Gamma)$ and the injection is continuous.

Lemma 4.3. Assume that $\lambda \in H_{0}^{-1 / 2}(\Gamma) \cap H_{\text {prod }}^{t}(\Gamma)$ for some $t>0$. Then there exists $\mu_{h} \in X_{h}^{0}$ such that

$$
\left\|\lambda-\mu_{h}\right\|_{-1 / 2, \Gamma} \leq C h^{\min \{t, m\}+1 / 2}\|\lambda\|_{t, \operatorname{prod}, \Gamma} .
$$

Here, $C>0$ is a constant independent of $h$.

Proof. Let $\mu_{h}$ be the best $L^{2}(\Gamma)$ approximation of $\lambda$ on $X_{h}$. Since constant functions belong to $X_{h}$, it follows that if $\lambda \in H_{0}^{-1 / 2}(\Gamma) \cap L^{2}(\Gamma)$, then $\mu_{h} \in X_{h}^{0}$. Notice also that, being $X_{h}$ a product of local spaces, $\mu_{h}$ is defined element by element. Using well-known arguments we can easily prove that

$$
\left\|\lambda-\mu_{h}\right\|_{0, \Gamma} \leq C h^{\min \{t, m\}}\|\lambda\|_{t, \operatorname{prod}, \Gamma} .
$$

Also, if $\xi \in H^{1}(\Gamma)$ and $\xi_{h}$ is its best $L^{2}(\Gamma)$ approximation on $X_{h}$ we have

$$
\left|\left\langle\lambda-\mu_{h}, \xi\right\rangle\right|=\left|\left\langle\lambda-\mu_{h}, \xi-\xi_{h}\right\rangle\right| \leq C h^{\min \{t, m\}+1}\|\lambda\|_{t, \text { prod, }, \Gamma}\|\xi\|_{1, \Gamma}
$$

and therefore

$$
\left\|\lambda-\mu_{h}\right\|_{-1, \Gamma} \leq C h^{\min \{t, m\}+1}\|\lambda\|_{t, \operatorname{prod}, \Gamma} .
$$

The result then follows by interpolation.

The a priori error estimate for the coupled LDG-BEM scheme (2.12) can be established now.

Theorem 4.1. Assume that $u \in H^{1+\delta}(\Omega)$ with $\delta>1 / 2$. Then there exists $C>0$, independent of $h$, such that

$$
\left\|\boldsymbol{\sigma}-\boldsymbol{\sigma}_{h}\right\|_{0, \Omega}+\left\|(u, \lambda)-\left(u_{h}, \lambda_{h}\right)\right\|_{h, \Gamma} \leq C h^{\min \{\delta, m\}}\|u\|_{1+\delta, \Omega} .
$$

Proof. It is not difficult to see that $u$ and $\lambda$ satisfy

$$
\begin{aligned}
B_{h}(u, v)+\langle\mathcal{W} u, v\rangle-\left\langle\left(\frac{1}{2} \mathcal{I}-\mathcal{K}^{\prime}\right) \lambda, v\right\rangle & =\int_{\Omega} f v, \\
\left\langle\mu,\left(\frac{1}{2} \mathcal{I}-\mathcal{K}\right) u\right\rangle+\langle\mu, \mathcal{V} \lambda\rangle & =0
\end{aligned}
$$

for any $(v, \mu) \in H_{1 / 2}^{1}\left(\mathcal{T}_{h}\right) \times H_{0}^{-1 / 2}(\Gamma)$. Using the bilinear form $\mathbf{B}_{h}$ (cf. (3.1)), the above means that

$$
\mathbf{B}_{h}(u, \lambda ; v, \mu)=\int_{\Omega} f v \quad \forall(v, \mu) \in H_{1 / 2}^{1}\left(\mathcal{T}_{h}\right) \times H^{-1 / 2}(\Gamma) .
$$

On the other hand, the discrete system (2.17) can be rewritten as

$$
\mathbf{B}_{h}\left(u_{h}, \lambda_{h} ; v, \mu\right)=\int_{\Omega} f v \quad \forall(v, \mu) \in \tilde{V}_{h} \times X_{h}^{0} .
$$

Hence, the ellipticity and continuity of the bilinear form $\mathbf{B}_{h}$ (cf. Lemma 3.1) imply the quasi-optimality

$$
\left\|(u, \lambda)-\left(u_{h}, \lambda_{h}\right)\right\|_{h, \Gamma} \leq C\left\|(u, \lambda)-\left(v_{h}, \mu_{h}\right)\right\|_{h, \Gamma} \quad \forall\left(v_{h}, \mu_{h}\right) \in \tilde{V}_{h} \times X_{h}^{0} .
$$


Also, since $\boldsymbol{\sigma}=\nabla u=\nabla u-\mathbf{S}_{h}(u)$ and $\boldsymbol{\sigma}_{h}=\nabla_{h} u_{h}-\mathbf{S}_{h}\left(u_{h}\right)$ (cf. Lemma 2.1), we obtain with (3.6) the upper bound

$$
\left\|\boldsymbol{\sigma}-\boldsymbol{\sigma}_{h}\right\|_{0, \Omega} \leq C\left\|u-u_{h}\right\|_{h},
$$

which, together with (4.15), gives

$$
\begin{array}{r}
\left\|\boldsymbol{\sigma}-\boldsymbol{\sigma}_{h}\right\|_{0, \Omega}+\left\|(u, \lambda)-\left(u_{h}, \lambda_{h}\right)\right\|_{h, \Gamma} \leq C\left\|(u, \lambda)-\left(v_{h}, \mu_{h}\right)\right\|_{h, \Gamma} \\
\forall\left(v_{h}, \mu_{h}\right) \in \tilde{V}_{h} \times X_{h}^{0} .
\end{array}
$$

Finally, applying the approximation properties from Lemmas 4.2 and 4.3 (with $t=\delta-1 / 2$ ), using (4.11) in the latter one, and combining the resulting estimates with (4.17) we arrive at (4.13). This finishes the proof.

We remark that the a priori error estimate (4.13) is independent of the polynomial degree $r$ that defines the subspace $\boldsymbol{\Sigma}_{h}$ (cf. (2.6) ). Hence, since the restriction $r \geq m-1$ is required only to deduce that $\boldsymbol{\sigma}_{h}=\nabla_{h} u_{h}-\mathbf{S}_{h}\left(u_{h}\right)$ (cf. Lemma 2.1), for practical computations it suffices to take $r=m-1$.

\section{The Coupled LDG-BEM scheme with Lagrangian Multiplier}

To implement the discrete scheme (2.12) one has to deal with the continuity condition of the space $\tilde{V}_{h}$. A direct implementation is possible without any difficulty. However, in order to maintain the full flexibility of the discontinuous method one can use a Lagrangian multiplier instead and work with $V_{h}$ rather than $\tilde{V}_{h}$. The needed multiplier is simply a vector of constants. In addition, the zero mean value condition of the unknown $\lambda_{h} \in X_{h}^{0}$ can be dealt with similarly, whence the resulting formulation employs the subspace $X_{h}$ instead of $X_{h}^{0}$. The description of this strategy and a simple numerical example illustrating its performance are provided in this section.

5.1. The Lagrangian multiplier approach. We first notice that the bilinear form of the coupled system (2.12), which is given by

$$
\begin{aligned}
\mathbf{A}_{h}(\boldsymbol{\zeta}, w, \xi ; \boldsymbol{\tau}, v, \mu):= & \int_{\Omega} \boldsymbol{\zeta} \cdot \boldsymbol{\tau}-\boldsymbol{\rho}(w, \boldsymbol{\tau})+\boldsymbol{\rho}(v, \boldsymbol{\zeta})+\boldsymbol{\alpha}(w, v)+\langle\mathcal{W} w, v\rangle \\
& -\left\langle\left(\frac{1}{2} \mathcal{I}-\mathcal{K}^{\prime}\right) \xi, v\right\rangle+\left\langle\mu,\left(\frac{1}{2} \mathcal{I}-\mathcal{K}\right) w\right\rangle+\langle\mu, \mathcal{V} \xi\rangle,
\end{aligned}
$$

is not well defined on $\Sigma_{h} \times V_{h} \times X_{h}$. For instance, the correct definition of the bilinear form $\langle\mathcal{W} w, v\rangle$ requires that $\left.w\right|_{\Gamma},\left.v\right|_{\Gamma} \in H^{1 / 2}(\Gamma)$. This is in general not true for $w, v \in V_{h}$. Therefore, we consider instead the bilinear form

$$
\begin{aligned}
\tilde{\mathbf{A}}_{h}(\boldsymbol{\zeta}, w, \xi ; \boldsymbol{\tau}, v, \mu):= & \int_{\Omega} \boldsymbol{\zeta} \cdot \boldsymbol{\tau}-\boldsymbol{\rho}(w, \boldsymbol{\tau})+\boldsymbol{\rho}(v, \boldsymbol{\zeta})+\boldsymbol{\alpha}(w, v)+\left\langle\partial_{h} w, \mathcal{V} \partial_{h} v\right\rangle \\
& -\left\langle\left(\frac{1}{2} \mathcal{I}-\mathcal{K}^{\prime}\right) \xi, v\right\rangle+\left\langle\mu,\left(\frac{1}{2} \mathcal{I}-\mathcal{K}\right) w\right\rangle+\langle\mu, \mathcal{V} \xi\rangle .
\end{aligned}
$$

Here, $\partial_{h} w$ is defined piecewise by $\left.\partial_{h} w\right|_{e}=\left(\left.w\right|_{e}\right)^{\prime}$ for any edge $e \in \Gamma_{h}$, and $\left(\left.w\right|_{e}\right)^{\prime}$ denotes the derivative of $w$ on $e$ with respect to the arc length. Note that $\partial_{h} w \in$ $L^{2}(\Gamma)$ for any $w \in V_{h}$. Then the updated bilinear form $\left\langle\partial_{h} w, \mathcal{V} \partial_{h} v\right\rangle$ is well defined for $w, v \in V_{h}$ and it holds that

$$
\langle\mathcal{W} w, v\rangle=\left\langle\partial_{h} w, \mathcal{V} \partial_{h} v\right\rangle \quad \forall w, v \in \tilde{V}_{h}
$$


(see [25]). Notice that the angled bracket in $\left\langle\mu,\left(\frac{1}{2} \mathcal{I}-\mathcal{K}\right) w\right\rangle$ is simply the $L^{2}(\Gamma)$ product and the term $\left(\frac{1}{2} \mathcal{I}-\mathcal{K}\right) w$ fails to be in $H^{1 / 2}(\Gamma)$ (it remains in $L^{2}(\Gamma)$ however), which is compensated, at this discrete level, by the fact that $\mu \in L^{2}(\Gamma)$. Therefore,

$\mathbf{A}_{h}(\boldsymbol{\zeta}, w, \xi ; \boldsymbol{\tau}, v, \mu)=\tilde{\mathbf{A}}_{h}(\boldsymbol{\zeta}, w, \xi ; \boldsymbol{\tau}, v, \mu) \quad \forall(\boldsymbol{\zeta}, w, \xi),(\boldsymbol{\tau}, v, \mu) \in \boldsymbol{\Sigma}_{h} \times \tilde{V}_{h} \times X_{h}$.

Now, let $\left\{\boldsymbol{z}_{1}, \ldots, \boldsymbol{z}_{n}\right\}$ denote the nodes of $\mathcal{T}_{h}$ on $\Gamma$ which belong to at least two triangles, and let $e_{i}^{-}$and $e_{i}^{+}$denote the two elements of $\Gamma_{h}$ which have $\boldsymbol{z}_{i}$ as a common node. The continuity of $u_{h}$ on $\Gamma$ is enforced through the equation

$$
\sum_{i=1}^{n}\left(\left.u_{h}\right|_{e_{i}^{+}}\left(\boldsymbol{z}_{i}\right)-\left.u_{h}\right|_{e_{i}^{-}}\left(\boldsymbol{z}_{i}\right)\right) y_{i}=0 \quad \forall\left(y_{1}, \ldots, y_{n}\right) \in \mathbb{R}^{n} .
$$

Similarly, the zero mean value condition of $\lambda_{h}$ is imposed as

$$
y_{n+1} \int_{\Gamma} \lambda_{h}=0 \quad \forall y_{n+1} \in \mathbb{R} .
$$

The above then suggests to define the bilinear form

$$
b_{h}((v, \mu), \overrightarrow{\mathbf{y}}):=\sum_{i=1}^{n}\left(\left.v\right|_{e_{i}^{+}}\left(\boldsymbol{z}_{i}\right)-\left.v\right|_{e_{i}^{-}}\left(\boldsymbol{z}_{i}\right)\right) y_{i}+y_{n+1} \int_{\Gamma} \mu
$$

for $(v, \mu) \in V_{h} \times X_{h}, \overrightarrow{\mathbf{y}}=\left(y_{1}, \ldots, y_{n+1}\right) \in \mathbb{R}^{n+1}$, and to consider the following LDG-BEM scheme with Lagrangian multiplier $\overrightarrow{\boldsymbol{x}}$ : Find $\left(\boldsymbol{\sigma}_{h}, u_{h}, \lambda_{h}, \overrightarrow{\boldsymbol{x}}\right) \in \boldsymbol{\Sigma}_{h} \times V_{h} \times$ $X_{h} \times \mathbb{R}^{n+1}$ such that

$$
\begin{aligned}
\tilde{\mathbf{A}}_{h}\left(\boldsymbol{\sigma}_{h}, u_{h}, \lambda_{h} ; \boldsymbol{\tau}, v, \mu\right)+b_{h}((v, \mu), \overrightarrow{\boldsymbol{x}}) & =\int_{\Omega} f v, \\
b_{h}\left(\left(u_{h}, \lambda_{h}\right), \overrightarrow{\mathbf{y}}\right) & =0
\end{aligned}
$$

for any $(\boldsymbol{\tau}, v, \mu, \overrightarrow{\mathbf{y}}) \in \boldsymbol{\Sigma}_{h} \times V_{h} \times X_{h} \times \mathbb{R}^{n+1}$. Then, we have the following result.

Theorem 5.1. There exists a unique solution $\left(\boldsymbol{\sigma}_{h}, u_{h}, \lambda_{h}, \overrightarrow{\boldsymbol{x}}\right) \in \boldsymbol{\Sigma}_{h} \times V_{h} \times X_{h} \times$ $\mathbb{R}^{n+1}$ of (5.2) and $\left(\boldsymbol{\sigma}_{h}, u_{h}, \lambda_{h}\right)$ solves (2.12). In particular, the error estimate from Theorem 4.1 holds.

Proof. It is immediate that there holds a (nonuniform) inf-sup condition for $b_{h}$ :

$$
\sup _{(v, \mu) \in V_{h} \times X_{h}} b_{h}((v, \mu), \overrightarrow{\mathbf{y}})>0 \quad \forall \overrightarrow{\mathbf{y}} \in \mathbb{R}^{n+1}, \quad \overrightarrow{\mathbf{y}} \neq 0 .
$$

We also have that the discrete null space of $b_{h}$ is given by

$$
\tilde{V}_{h} \times X_{h}^{0}=\left\{(v, \mu) \in V_{h} \times X_{h}: \quad b_{h}((v, \mu), \overrightarrow{\mathbf{y}})=0 \quad \forall \overrightarrow{\mathbf{y}} \in \mathbb{R}^{n+1}\right\} .
$$

Therefore, Theorem 3.1 and the Babuška-Brezzi theory for discrete problems ensure the unique solvability of (5.2) and then $\left(\boldsymbol{\sigma}_{h}, u_{h}, \lambda_{h}\right) \in \boldsymbol{\Sigma}_{h} \times \tilde{V}_{h} \times X_{h}^{0}$ becomes the unique solution of (2.12), whence the error estimate of Theorem 4.1 holds.

5.2. A numerical example. In this section we present a simple numerical example illustrating the performance of (5.2) with $m=1$ and $r=m-1=0$. This means, according to (2.6) and (2.11), that

$$
\boldsymbol{\Sigma}_{h}:=\prod_{K \in \mathcal{T}_{h}} \mathbf{P}_{0}(K), \quad V_{h}:=\prod_{K \in \mathcal{T}_{h}} P_{1}(K), \quad \text { and } \quad X_{h}:=\prod_{e \in \mathcal{E}_{h}^{\Gamma}} P_{0}(e) .
$$

In this case, as established by Theorem 4.1 with $\delta=1$, for a continuous solution $u \in H^{2}(\Omega)$ there holds a rate of convergence of $O(h)$. 
TABLE 1. Degrees of freedom, meshsizes, errors, and rates of convergence

\begin{tabular}{r|c||c|c||c|c||c|c}
$N$ & $h$ & $\mathrm{e}(\boldsymbol{\sigma})$ & $\mathrm{r}(\boldsymbol{\sigma})$ & $\mathrm{e}(u)$ & $\mathrm{r}(u)$ & $\mathrm{e}(\lambda)$ & $\mathrm{r}(\lambda)$ \\
\hline 54 & 0.50000 & $0.4053 \mathrm{E}+00$ & - & $0.1714 \mathrm{E}+01$ & - & $0.3269 \mathrm{E}+01$ & - \\
112 & 0.33333 & $0.2596 \mathrm{E}+00$ & 1.098 & $0.1246 \mathrm{E}+01$ & 0.786 & $0.2345 \mathrm{E}+01$ & 0.819 \\
190 & 0.25000 & $0.1871 \mathrm{E}+00$ & 1.139 & $0.9307 \mathrm{E}+00$ & 1.014 & $0.1794 \mathrm{E}+01$ & 0.931 \\
288 & 0.20000 & $0.1464 \mathrm{E}+00$ & 1.099 & $0.7667 \mathrm{E}+00$ & 0.869 & $0.1419 \mathrm{E}+01$ & 1.051 \\
406 & 0.16667 & $0.1203 \mathrm{E}+00$ & 1.077 & $0.6530 \mathrm{E}+00$ & 0.881 & $0.1171 \mathrm{E}+01$ & 1.054 \\
544 & 0.14286 & $0.1023 \mathrm{E}+00$ & 1.051 & $0.5678 \mathrm{E}+00$ & 0.907 & $0.9979 \mathrm{E}+00$ & 1.038 \\
702 & 0.12500 & $0.8898 \mathrm{E}-01$ & 1.045 & $0.5021 \mathrm{E}+00$ & 0.921 & $0.8699 \mathrm{E}+00$ & 1.028 \\
880 & 0.11111 & $0.7870 \mathrm{E}-01$ & 1.042 & $0.4500 \mathrm{E}+00$ & 0.930 & $0.7713 \mathrm{E}+00$ & 1.021 \\
1078 & 0.10000 & $0.7054 \mathrm{E}-01$ & 1.039 & $0.4077 \mathrm{E}+00$ & 0.937 & $0.6928 \mathrm{E}+00$ & 1.019 \\
1296 & 0.09091 & $0.6391 \mathrm{E}-01$ & 1.036 & $0.3727 \mathrm{E}+00$ & 0.942 & $0.6290 \mathrm{E}+00$ & 1.014 \\
1534 & 0.08333 & $0.5842 \mathrm{E}-01$ & 1.032 & $0.3431 \mathrm{E}+00$ & 0.951 & $0.5760 \mathrm{E}+00$ & 1.011 \\
1792 & 0.07692 & $0.5379 \mathrm{E}-01$ & 1.032 & $0.3179 \mathrm{E}+00$ & 0.953 & $0.5313 \mathrm{E}+00$ & 1.009 \\
2070 & 0.07143 & $0.4984 \mathrm{E}-01$ & 1.030 & $0.2962 \mathrm{E}+00$ & 0.955 & $0.4932 \mathrm{E}+00$ & 1.005
\end{tabular}

We now describe the example. We consider a slightly simplified model with no interior region $\Omega_{0}$, take $\left.\Omega=\right] 0,1\left[^{2}\right.$, and choose the data so that the exact solution is given by

$$
u(\mathbf{x})=x_{1}^{2}+x_{2}^{2} \quad \forall \mathbf{x}:=\left(x_{1}, x_{2}\right)^{\mathrm{t}} \in \Omega
$$

and

$$
u_{e}(\mathbf{x})=\frac{x_{1}+x_{2}-1}{\left(x_{1}-0.5\right)^{2}+\left(x_{2}-0.5\right)^{2}} \quad \forall \mathbf{x}:=\left(x_{1}, x_{2}\right)^{\mathrm{t}} \in \Omega_{e} .
$$

Since $u$ and $u_{e}$ do not coincide on $\Gamma:=\partial \Omega$, we need to allow for nonhomogeneous transmission conditions, which means replacing (1.4) by

(5.3) $u-u_{e}=g_{0} \in H^{1 / 2}(\Gamma)$ on $\Gamma \quad$ and $\partial_{\nu} u-\partial_{\nu} u_{e}=g_{1} \in L^{2}(\Gamma)$ on $\Gamma$.

Note here that the smoother assumption on $g_{1}$ is required to be able to introduce the function $\lambda_{h}+g_{1}$ in the LDG module (2.8) as the suitable $L^{2}(\Gamma)$ approximation of the normal derivative $\partial_{\boldsymbol{\nu}} u$ on $\Gamma$. In this case without interior Dirichlet boundary $\Gamma_{0}$ one finds that $\int_{\Gamma} \lambda_{h}=0$ is automatically satisfied (choose $(\tau, v, \mu)=(0,1,0)$ in (5.2) and make use of the relation $\mathcal{K} 1=-1 / 2)$. We therefore use, instead of the bilinear form $b_{h}$ defined by (5.1), the reduced form

$$
\tilde{b}_{h}((v, \mu), \overrightarrow{\mathbf{y}}):=\sum_{i=1}^{n}\left(\left.v\right|_{e_{i}^{+}}\left(\boldsymbol{z}_{i}\right)-\left.v\right|_{e_{i}^{-}}\left(\boldsymbol{z}_{i}\right)\right) y_{i}
$$

for $(v, \mu) \in V_{h} \times X_{h}, \overrightarrow{\mathbf{y}}=\left(y_{1}, \ldots, y_{n}\right) \in \mathbb{R}^{n}$. As a consequence, the scheme (5.2) becomes: Find $\left(\boldsymbol{\sigma}_{h}, u_{h}, \lambda_{h}, \overrightarrow{\boldsymbol{x}}\right) \in \boldsymbol{\Sigma}_{h} \times V_{h} \times X_{h} \times \mathbb{R}^{n}$ such that

$$
\begin{aligned}
\tilde{\mathbf{A}}_{h}\left(\boldsymbol{\sigma}_{h}, u_{h}, \lambda_{h} ; \boldsymbol{\tau}, v, \mu\right)+\tilde{b}_{h}((v, \mu), \overrightarrow{\boldsymbol{x}}) & =F(v, \mu), \\
\tilde{b}_{h}\left(\left(u_{h}, \lambda_{h}\right), \overrightarrow{\mathbf{y}}\right) & =0
\end{aligned}
$$

for any $(\boldsymbol{\tau}, v, \mu, \overrightarrow{\mathbf{y}}) \in \boldsymbol{\Sigma}_{h} \times V_{h} \times X_{h} \times \mathbb{R}^{n}$, where

$$
F(v, \mu):=\int_{\Omega} f v+\int_{\Gamma} g_{1} v+\left\langle\partial_{h} g_{0}, \mathcal{V} \partial_{h} v\right\rangle+\left\langle\mu,\left(\frac{1}{2}-\mathcal{K}\right) g_{0}\right\rangle .
$$




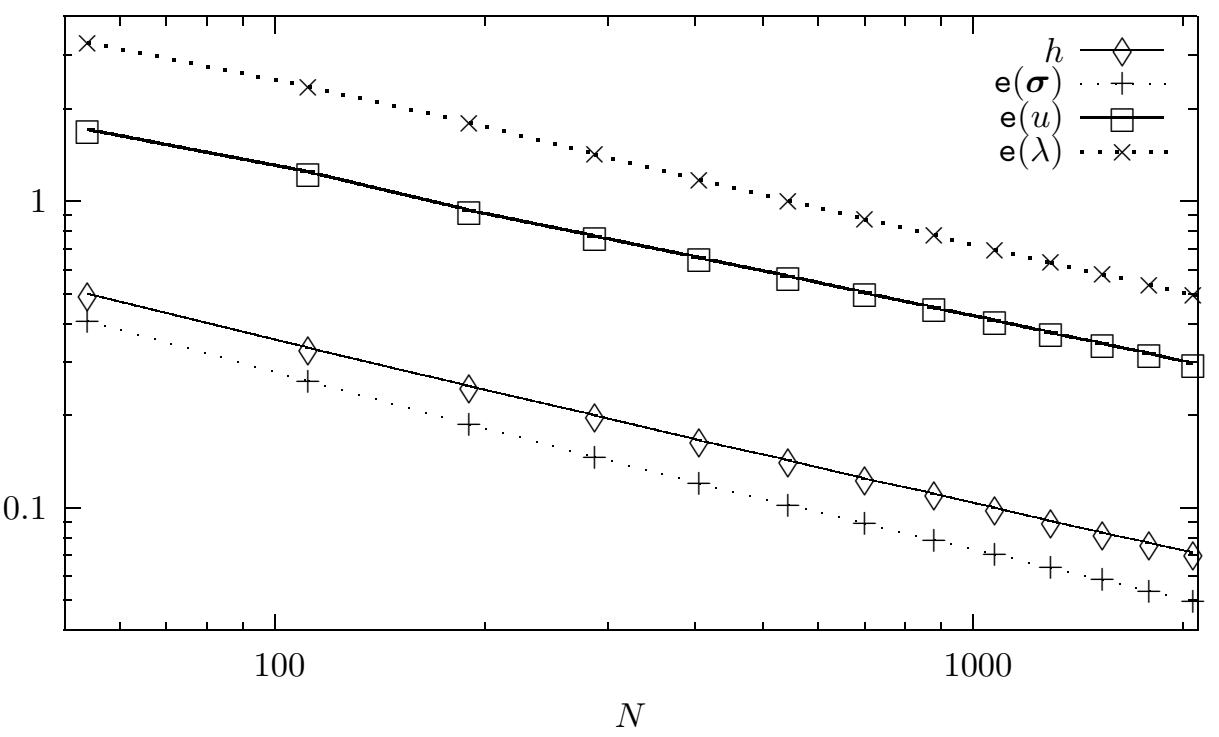

FiguRE 2. meshsize $h$ and errors vs. degrees of freedom $N$

The numerical results shown below were obtained using a Fortran implementation. In what follows the variable $N$ stands for the number of degrees of freedom defining $\boldsymbol{\Sigma}_{h}, V_{h}, X_{h}$, and $\mathbb{R}^{n}$, and the individual errors are denoted by

$$
\begin{aligned}
& \mathrm{e}(\boldsymbol{\sigma}):=\left\|\boldsymbol{\sigma}-\boldsymbol{\sigma}_{h}\right\|_{0, \Omega}, \\
& \mathrm{e}(u):=\left\{\left\|u-u_{h}\right\|_{h}^{2}+\left\|u-u_{h}\right\|_{0, \Gamma}\left|u-u_{h}\right|_{1, \Gamma}\right\}^{1 / 2}, \\
& \mathrm{e}(\lambda)=\left\|\lambda-\lambda_{h}\right\|_{0, \Gamma} .
\end{aligned}
$$

By interpolation, $\left\|u-u_{h}\right\|_{0, \Gamma}\left|u-u_{h}\right|_{1, \Gamma}$ is an upper bound for $\left\|u-u_{h}\right\|_{1 / 2, \Gamma, 0}^{2}$, and $\mathrm{e}(\lambda)$ is an upper bound for $\left\|\lambda-\lambda_{h}\right\|_{-1 / 2, \Gamma}$. Presenting the errors $\mathrm{e}(\boldsymbol{\sigma})$, e $(u)$ and $\mathrm{e}(\lambda)$ is therefore sufficient to verify the a priori error estimate given by Theorem 4.1 . by

Also, we let $r(\boldsymbol{\sigma}), r(u)$, and $r(\lambda)$ be the experimental rates of convergence given

$$
\begin{aligned}
& r(\boldsymbol{\sigma}):=\frac{\log \left(\mathrm{e}(\boldsymbol{\sigma}) / \mathrm{e}^{\prime}(\boldsymbol{\sigma})\right)}{\log \left(h / h^{\prime}\right)}, \\
& r(u):=\frac{\log \left(\mathrm{e}(u) / \mathrm{e}^{\prime}(u)\right)}{\log \left(h / h^{\prime}\right)}, \\
& r(\lambda):=\frac{\log \left(\mathrm{e}(\lambda) / \mathrm{e}^{\prime}(\lambda)\right)}{\log \left(h / h^{\prime}\right)},
\end{aligned}
$$

where $h$ and $h^{\prime}$ denote two consecutive meshsizes with errors e and $\mathrm{e}^{\prime}$.

In Table 1 we present the convergence history of the example for a set of uniform triangulations of the computational domain $\Omega \cup \Gamma$. A rate of convergence $O(h)$ is attained by all the unknowns and this confirms the error estimate by Theorem 4.1 . The dominant error is given by $\mathrm{e}(\lambda)$ which, being measured in the $L^{2}(\Gamma)$-norm, overestimates the error $\left\|\lambda-\lambda_{h}\right\|_{-1 / 2, \Gamma}$ but confirms the convergence like $O(h)$. 
The experimental rates of convergence and the dominant component of the error can also be checked from Figure 2 where we display the meshsize $h$ and the errors $\mathrm{e}(\boldsymbol{\sigma}), \mathrm{e}(u)$, and $\mathrm{e}(\lambda)$ vs. the degrees of freedom $N$.

We end this section by remarking that the same results are obtained by implementing the continuity on $\Gamma$ for the functions in $\tilde{V}_{h}$ and then solving the original coupled LDG-BEM scheme (2.12) instead of (5.2) (15.4) in this case). In addition, similar results and the same rate of convergence $O(h)$ are obtained with the mortar-based coupling scheme from [18. In this respect we emphasize again that the present approach is computationally appealing since the number of unknown functions is reduced by two while only standard LDG (and BEM) discretizations are needed when using the Lagrangian multiplier.

\section{EXTENSION TO NONLINEAR PROBLEMS}

In this section we extend the present LDG-BEM approach to the class of nonlinear exterior transmission problems studied in [9, [10, and [11. In order to describe the model problem let $\Omega_{0}$ be a simply connected and bounded domain in $\mathbb{R}^{2}$ with polygonal boundary $\Gamma_{0}$. Then, let $\Omega_{1}$ be an annular and simply connected domain surrounded by $\Gamma_{0}$ and another polygonal boundary $\Gamma_{1}$. In addition, let a : $\Omega_{1} \times \mathbb{R}^{2} \rightarrow \mathbb{R}^{2}$ be a nonlinear function satisfying the conditions specified in 7] (see also [9]) which, in particular, imply that the associated operator becomes Lipschitz continuous and strongly monotone. Thus, given $f \in L^{2}\left(\mathbb{R}^{2} \backslash \bar{\Omega}_{0}\right)$ with compact support, $g_{0} \in H^{1 / 2}\left(\Gamma_{0}\right), g_{1} \in H^{1 / 2}\left(\Gamma_{1}\right)$, and $g_{2} \in L^{2}\left(\Gamma_{1}\right)$, we consider the nonlinear exterior transmission problem:

$$
\begin{gathered}
-\operatorname{div} \mathbf{a}\left(\cdot, \nabla u_{1}\right)=f \quad \text { in } \quad \Omega_{1}, \quad u_{1}=g_{0} \quad \text { on } \Gamma_{0}, \\
-\Delta u_{2}=f \quad \text { in } \quad \mathbb{R}^{2} \backslash\left(\bar{\Omega}_{0} \cup \bar{\Omega}_{1}\right), \quad u_{1}-u_{2}=g_{1} \quad \text { on } \Gamma_{1}, \\
\mathbf{a}\left(\cdot, \nabla u_{1}\right) \cdot \boldsymbol{\nu}_{1}-\nabla u_{2} \cdot \boldsymbol{\nu}_{1}=g_{2} \quad \text { on } \quad \Gamma_{1}, \quad \text { and } \quad u_{2}(\boldsymbol{x})=\mathcal{O}(1) \quad \text { as } \quad|\boldsymbol{x}| \rightarrow \infty .
\end{gathered}
$$

Here, $\boldsymbol{\nu}_{1}$ stands for the unit outward normal to $\Gamma_{1}$. This kind of problems appears in the computation of magnetic fields of electromagnetic devices (see, e.g. 21, 22]), in some subsonic flow and fluid mechanics problems (see, e.g. [16], [17]), and also in steady state heat conduction. For instance, in the latter case, one has $\mathbf{a}(\boldsymbol{x}, \nabla u(\boldsymbol{x}))=k(\boldsymbol{x}, \nabla u(\boldsymbol{x})) \nabla u$, where $u$ is the temperature and $k: \Omega_{1} \times \mathbb{R}^{2} \rightarrow \mathbb{R}$ is the heat conductivity.

Next, we introduce a closed polygonal curve $\Gamma$ such that its interior contains the support of $f$. Then, let $\Omega_{2}$ be the annular domain bounded by $\Gamma_{1}$ and $\Gamma$ and set $\Omega_{e}:=\mathbb{R}^{2} \backslash\left(\bar{\Omega}_{0} \cup \bar{\Omega}_{1} \cup \bar{\Omega}_{2}\right)$ (see Figure 3 below). It follows that (6.1) can be equivalently rewritten as the nonlinear boundary value problem in $\Omega_{1}$ :

$$
-\operatorname{div} \mathbf{a}\left(\cdot, \nabla u_{1}\right)=f \quad \text { in } \quad \Omega_{1}, \quad u_{1}=g_{0} \quad \text { on } \Gamma_{0},
$$

the Poisson equation in $\Omega_{2}$ :

$$
-\Delta u_{2}=f \quad \text { in } \quad \Omega_{2}
$$

and the Laplace equation in the exterior unbounded region $\Omega_{e}$ :

$$
-\Delta u_{2}=0 \quad \text { in } \quad \Omega_{e}, \quad u_{2}(\boldsymbol{x})=\mathcal{O}(1) \quad \text { as } \quad|\boldsymbol{x}| \rightarrow \infty,
$$




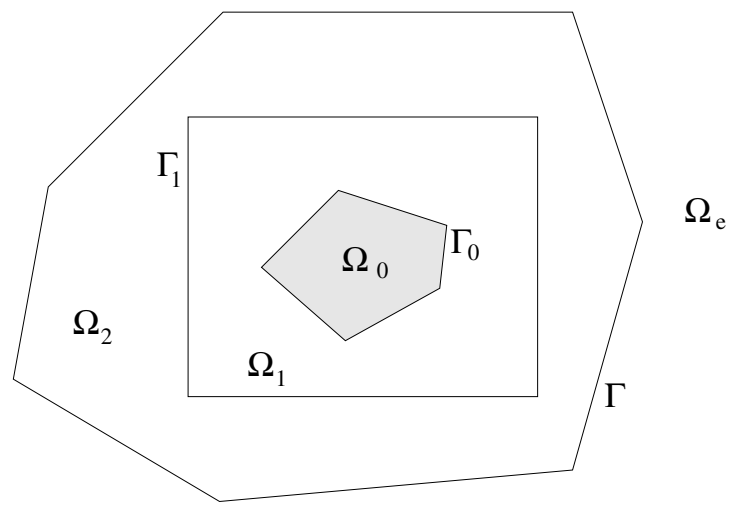

FiguRE 3. Geometry of the transmission problem.

coupled with the transmission conditions on $\Gamma_{1}$ and $\Gamma$, respectively,

$$
u_{1}-u_{2}=g_{1} \quad \text { and } \quad \mathbf{a}\left(\cdot, \nabla u_{1}\right) \cdot \boldsymbol{\nu}_{1}-\nabla u_{2} \cdot \boldsymbol{\nu}_{1}=g_{2} \text { on } \Gamma_{1},
$$

and

(6.6)

$$
\lim _{\substack{\boldsymbol{x} \rightarrow \boldsymbol{x}_{0} \\ \boldsymbol{x} \in \Omega_{2}}} u_{2}(\boldsymbol{x})=\lim _{\substack{\boldsymbol{x} \rightarrow \boldsymbol{x}_{0} \\ \boldsymbol{x} \in \Omega_{e}}} u_{2}(\boldsymbol{x}) \text { and } \lim _{\substack{\boldsymbol{x} \rightarrow \boldsymbol{x}_{0} \\ \boldsymbol{x} \in \Omega_{2}}} \nabla u_{2}(\boldsymbol{x}) \cdot \boldsymbol{\nu}\left(\boldsymbol{x}_{0}\right)=\lim _{\substack{\boldsymbol{x} \rightarrow \boldsymbol{x}_{0} \\ \boldsymbol{x} \in \Omega_{e}}} \nabla u_{2}(\boldsymbol{x}) \cdot \boldsymbol{\nu}\left(\boldsymbol{x}_{0}\right)
$$

for almost all $\boldsymbol{x}_{0} \in \Gamma$, where $\boldsymbol{\nu}\left(\boldsymbol{x}_{0}\right)$ denotes the unit outward normal to $\boldsymbol{x}_{0}$.

We now follow [18 and 9] and introduce the gradients $\boldsymbol{\theta}_{1}:=\nabla u_{1}$ in $\Omega_{1}$ and $\boldsymbol{\theta}_{2}:=\nabla u_{2}$ in $\Omega_{2}$, and the fluxes $\boldsymbol{\sigma}_{1}:=\mathbf{a}\left(\cdot, \boldsymbol{\theta}_{1}\right)$ in $\Omega_{1}$ and $\boldsymbol{\sigma}_{2}:=\boldsymbol{\theta}_{2}$ in $\Omega_{2}$, as additional unknowns. Also, as in Section 2, let $\lambda_{h} \in X_{h}^{0}$ be a discrete approximation of the normal derivative $\lambda:=\partial_{\boldsymbol{\nu}} u_{2}$ on $\Gamma$, and proceeding in the usual way (see 9] for details). We arrive at the following global LDG formulation in $\Omega:=\Omega_{1} \cup \Gamma_{1} \cup \Omega_{2}$ : Find $\left(\boldsymbol{\theta}_{h}, \boldsymbol{\sigma}_{h}, u_{h}\right) \in \boldsymbol{\Sigma}_{h} \times \boldsymbol{\Sigma}_{h} \times \tilde{V}_{h}$ such that

$$
\begin{array}{ccrl}
\int_{\Omega} \overline{\mathbf{a}}\left(\cdot, \boldsymbol{\theta}_{h}\right) \cdot \boldsymbol{\zeta}-\int_{\Omega} \boldsymbol{\sigma}_{h} \cdot \boldsymbol{\zeta} & =0 & & \forall \boldsymbol{\zeta} \in \boldsymbol{\Sigma}_{h}, \\
\int_{\Omega} \boldsymbol{\theta}_{h} \cdot \boldsymbol{\tau}-\left\{\int_{\Omega} \nabla_{h} u_{h} \cdot \boldsymbol{\tau}-S\left(u_{h}, \boldsymbol{\tau}\right)\right\} & =G_{h}(\boldsymbol{\tau}) & & \forall \boldsymbol{\tau} \in \boldsymbol{\Sigma}_{h}, \\
\left\{\int_{\Omega} \nabla_{h} v \cdot \boldsymbol{\sigma}_{h}-S\left(v, \boldsymbol{\sigma}_{h}\right)\right\}+\boldsymbol{\alpha}\left(u_{h}, v\right) & =F_{h}(v)+\int_{\Gamma} \lambda_{h} v & & \forall v \in \tilde{V}_{h},
\end{array}
$$

where

$$
\overline{\mathbf{a}}(\cdot, \boldsymbol{\zeta}):=\left\{\begin{array}{cc}
\mathbf{a}(\cdot, \boldsymbol{\zeta}) & \text { in } \Omega_{1} \\
\boldsymbol{\zeta} & \text { in } \Omega_{2}
\end{array} \quad \forall \boldsymbol{\zeta} \in\left[L^{2}(\Omega)\right]^{2}\right.
$$


and the bilinear forms $S: H^{1}\left(\mathcal{T}_{h}\right) \times \boldsymbol{L}^{2}(\Omega) \rightarrow \mathbb{R}$ and $\boldsymbol{\alpha}: H^{1}\left(\mathcal{T}_{h}\right) \times H^{1}\left(\mathcal{T}_{h}\right) \rightarrow \mathbb{R}$ as well as the linear operators $G_{h}: \boldsymbol{L}^{2}(\Omega) \rightarrow \mathbb{R}$ and $F_{h}: H^{1}\left(\mathcal{T}_{h}\right) \rightarrow \mathbb{R}$ are defined by

$$
\begin{gathered}
S(w, \boldsymbol{\tau}):=\int_{I_{h}} \llbracket w \rrbracket \cdot(\{\boldsymbol{\tau}\}-\llbracket \boldsymbol{\tau} \rrbracket \boldsymbol{\beta})+\int_{\Gamma_{0}} w\left(\boldsymbol{\tau}_{1} \cdot \boldsymbol{\nu}\right)+\int_{\Gamma_{1}}\left(w_{1}-w_{2}\right) \boldsymbol{\tau}_{1} \cdot \boldsymbol{\nu}_{1}, \\
\boldsymbol{\alpha}(w, v):=\int_{I_{h}} \alpha \llbracket w \rrbracket \cdot \llbracket v \rrbracket+\int_{\Gamma_{0}} \alpha w v+\int_{\Gamma_{1}} \alpha\left(w_{1}-w_{2}\right)\left(v_{1}-v_{2}\right), \\
G_{h}(\boldsymbol{\tau}):=\int_{\Gamma_{0}} g_{0} \boldsymbol{\tau}_{1} \cdot \boldsymbol{\nu}+\int_{\Gamma_{1}} g_{1} \boldsymbol{\tau}_{1} \cdot \boldsymbol{\nu}_{1},
\end{gathered}
$$

and

$$
F_{h}(v):=\int_{\Omega} f v+\int_{\Gamma_{0}} \alpha g_{0} v_{1}+\int_{\Gamma_{1}} \alpha g_{1}\left(v_{1}-v_{2}\right)+\int_{\Gamma_{1}} g_{2} v_{2}
$$

for all $w, v \in H^{1}\left(\mathcal{T}_{h}\right), \boldsymbol{\tau} \in \boldsymbol{L}^{2}(\Omega)$, with $w_{i}:=\left.w\right|_{\Omega_{i}}, v_{i}:=\left.v\right|_{\Omega_{i}}$, and $\boldsymbol{\tau}_{i}:=\left.\boldsymbol{\tau}\right|_{\Omega_{i}}$, for each $i \in\{1,2\}$. Hereafter, $\mathcal{T}_{h}=\mathcal{T}_{h, 1} \cup \mathcal{T}_{h, 2}$, where $\mathcal{T}_{h, 1}$ and $\mathcal{T}_{h, 2}$ are shape regular triangulations of $\bar{\Omega}_{1}$ and $\bar{\Omega}_{2}$, respectively, which satisfy the same properties and assumptions as indicated in Section 2.2

Next, introducing the boundary integral formulation in $\Omega_{e}$, exactly as in Section 2.1. substituting $\lambda_{h}$ in (6.7) by a discrete version of the first equation in (2.2), in which $u$ is replaced by its approximant $u_{h}$, and adding a discrete formulation of the second equation in (2.2), we obtain the following coupled LDG-BEM scheme: Find $\left(\boldsymbol{\theta}_{h}, \boldsymbol{\sigma}_{h}, u_{h}, \lambda_{h}\right) \in \boldsymbol{\Sigma}_{h} \times \boldsymbol{\Sigma}_{h} \times \tilde{V}_{h} \times X_{h}^{0}$ such that

$$
\begin{aligned}
\int_{\Omega} \overline{\mathbf{a}}\left(\cdot, \boldsymbol{\theta}_{h}\right) \cdot \boldsymbol{\zeta}-\int_{\Omega} \boldsymbol{\sigma}_{h} \cdot \boldsymbol{\zeta} & =0, \\
\int_{\Omega} \boldsymbol{\theta}_{h} \cdot \boldsymbol{\tau}-\boldsymbol{\rho}\left(u_{h}, \boldsymbol{\tau}\right) & =G_{h}(\boldsymbol{\tau}), \\
\boldsymbol{\rho}\left(v, \boldsymbol{\sigma}_{h}\right)+\boldsymbol{\alpha}\left(u_{h}, v\right)+\left\langle\mathcal{W} u_{h}, v\right\rangle-\left\langle\left(\frac{1}{2} \mathcal{I}-\mathcal{K}^{\prime}\right) \lambda_{h}, v\right\rangle & =F_{h}(v), \\
\left\langle\mu,\left(\frac{1}{2} \mathcal{I}-\mathcal{K}\right) u_{h}\right\rangle+\left\langle\mu, \mathcal{V} \lambda_{h}\right\rangle & =0
\end{aligned}
$$

for all $(\boldsymbol{\zeta}, \boldsymbol{\tau}, v, \mu) \in \boldsymbol{\Sigma}_{h} \times \boldsymbol{\Sigma}_{h} \times \tilde{V}_{h} \times X_{h}^{0}$, where $\boldsymbol{\rho}: H^{1}\left(\mathcal{T}_{h}\right) \times \mathbf{H}^{1}\left(\mathcal{T}_{h}\right) \rightarrow \mathbb{R}$ is the analogue of the bilinear form defined by (2.13); that is,

$$
\boldsymbol{\rho}(v, \boldsymbol{\tau}):=\int_{\Omega} \nabla_{h} v \cdot \boldsymbol{\tau}-S(v, \boldsymbol{\tau}) \quad \forall(v, \boldsymbol{\tau}) \in H^{1}\left(\mathcal{T}_{h}\right) \times \mathbf{H}^{1}\left(\mathcal{T}_{h}\right) .
$$

In what follows we proceed as in Section [2.3 (see also Section 2.4 of [18]) and derive an equivalent formulation to (6.8). We begin by defining a linear operator $\mathbf{S}_{h}: H^{1}\left(\mathcal{T}_{h}\right) \rightarrow \boldsymbol{\Sigma}_{h}$ as in (2.15), where, given $v \in H^{1}\left(\mathcal{T}_{h}\right), \mathbf{S}_{h}(v)$ is the unique element in $\boldsymbol{\Sigma}_{h}$ such that

$$
\int_{\Omega} \mathbf{S}_{h}(v) \cdot \boldsymbol{\tau}=S(v, \boldsymbol{\tau}) \quad \forall \boldsymbol{\tau} \in \mathbf{\Sigma}_{h}
$$

Next, let $\mathcal{G}_{h}$ be the unique element in $\boldsymbol{\Sigma}_{h}$ such that

$$
\int_{\Omega} \mathcal{G}_{h} \cdot \boldsymbol{\tau}=G_{h}(\boldsymbol{\tau}):=\int_{\Gamma_{0}} g_{0} \boldsymbol{\tau}_{1} \cdot \boldsymbol{\nu}+\int_{\Gamma_{1}} g_{1} \boldsymbol{\tau}_{1} \cdot \boldsymbol{\nu}_{1} \quad \forall \boldsymbol{\tau} \in \boldsymbol{\Sigma}_{h} .
$$


It is easy to see that $\left.\mathcal{G}_{h}\right|_{\Omega_{2}}=\mathbf{0}$. From now on we set

$$
u:=\left\{\begin{array}{l}
u_{1} \text { in } \Omega_{1} \\
u_{2} \text { in } \Omega_{2}
\end{array}\right.
$$

Then, if the solution of problem (6.1) satisfies $u_{1} \in H^{t}\left(\Omega_{1}\right)$ and $u_{2} \in H^{s}\left(\Omega_{2}\right)$, with $t, s>1$, we find that $\mathbf{S}_{h}(u)=\mathcal{G}_{h}$. In addition, it follows from the first two equations in (6.8) that, whenever this system is solvable, it holds that

$$
\boldsymbol{\theta}_{h}=\nabla_{h} u_{h}-\mathbf{S}_{h}\left(u_{h}\right)+\mathcal{G}_{h} \quad \text { and } \quad \boldsymbol{\sigma}_{h}=\Pi_{\boldsymbol{\Sigma}_{h}} \overline{\mathbf{a}}\left(\cdot, \boldsymbol{\theta}_{h}\right),
$$

where $\Pi_{\boldsymbol{\Sigma}_{h}}$ denotes the $\boldsymbol{L}^{2}(\Omega)$-orthogonal projection onto $\boldsymbol{\Sigma}_{h}$. We observe here, as in the proof of Lemma 2.1, that the fact that $r \geq m-1$ guarantees that $\nabla_{h} u_{h} \in \boldsymbol{\Sigma}_{h}$, which yields the above expression for $\boldsymbol{\theta}_{h}$. Then, replacing the unknown $\boldsymbol{\sigma}_{h}$ by

$$
\Pi_{\boldsymbol{\Sigma}_{h}} \overline{\mathbf{a}}\left(\cdot, \nabla_{h} u_{h}-\mathbf{S}_{h}\left(u_{h}\right)+\mathcal{G}_{h}\right)
$$

in the third equation of (6.8), we are led to the semilinear form $A_{h}: H^{1}\left(\mathcal{T}_{h}\right) \times$ $H^{1}\left(\mathcal{T}_{h}\right) \rightarrow \mathbb{R}$ defined by

$A_{h}(w, v):=\boldsymbol{\alpha}(w, v)+\int_{\Omega} \overline{\mathbf{a}}\left(\cdot, \nabla_{h} w-\mathbf{S}_{h}(w)+\mathcal{G}_{h}\right) \cdot\left(\nabla_{h} v-\mathbf{S}_{h}(v)\right) \quad \forall w, v \in H^{1}\left(\mathcal{T}_{h}\right)$.

Moreover, we can establish the following equivalence result which constitutes the nonlinear analogue of Lemma 2.1.

Lemma 6.1. Let $\left(\boldsymbol{\theta}_{h}, \boldsymbol{\sigma}_{h}, u_{h}, \lambda_{h}\right) \in \boldsymbol{\Sigma}_{h} \times \boldsymbol{\Sigma}_{h} \times \tilde{V}_{h} \times X_{h}^{0}$ be a solution of (6.8). Then it holds that

$$
\begin{aligned}
A_{h}\left(u_{h}, v\right)+\left\langle\mathcal{W} u_{h}, v\right\rangle-\left\langle\left(\frac{1}{2} \mathcal{I}-\mathcal{K}^{\prime}\right) \lambda_{h}, v\right\rangle & =F_{h}(v), \\
\left\langle\mu,\left(\frac{1}{2} \mathcal{I}-\mathcal{K}\right) u_{h}\right\rangle+\left\langle\mu, \mathcal{V} \lambda_{h}\right\rangle & =0
\end{aligned}
$$

for any $(v, \mu) \in \tilde{V}_{h} \times X_{h}^{0}$. Conversely, if $\left(u_{h}, \lambda_{h}\right) \in \tilde{V}_{h} \times X_{h}^{0}$ satisfies (6.12) and $\boldsymbol{\theta}_{h}$ and $\boldsymbol{\sigma}_{h}$ are defined by (6.11), then $\left(\boldsymbol{\theta}_{h}, \boldsymbol{\sigma}_{h}, u_{h}, \lambda_{h}\right)$ is a solution of (6.8). If $\left(u_{h}, \lambda_{h}\right) \in \tilde{V}_{h} \times X_{h}^{0}$ is the only solution of (6.12), then $\left(\boldsymbol{\theta}_{h}, \boldsymbol{\sigma}_{h}, u_{h}, \lambda_{h}\right)$, with $\boldsymbol{\theta}_{h}$ and $\boldsymbol{\sigma}_{h}$ defined as indicated above, is the only solution of (6.8).

Proof. It is similar to the proof of Lemma 2.1 (see also Lemma 2.2 in [18]) and is based on the identities (6.11).

We now introduce seminorms

$$
\begin{aligned}
|v|_{1, h}^{2}:=\left\|\nabla_{h} v\right\|_{0, \Omega}^{2}, \quad|v|_{*}^{2}:=\left\|h_{\mathcal{E}}^{-1 / 2} \llbracket v \rrbracket\right\|_{0, I_{h}}^{2}+\left\|h_{\mathcal{E}}^{-1 / 2} v\right\|_{0, \Gamma_{0}}^{2}+\left\|h_{\mathcal{E}}^{-1 / 2}\left(v_{1}-v_{2}\right)\right\|_{0, \Gamma_{1}}^{2} \\
\forall v \in H^{1}\left(\mathcal{T}_{h}\right),
\end{aligned}
$$

and the norms

$$
\begin{array}{r}
\|v\|_{h}^{2}:=|v|_{1, h}^{2}+|v|_{*}^{2} \quad \forall v \in H^{1}\left(\mathcal{T}_{h}\right), \\
\|(v, \mu)\|_{h, \Gamma}^{2}:=\|v\|_{h}^{2}+\|v\|_{1 / 2, \Gamma, 0}^{2}+\|\mu\|_{-1 / 2, \Gamma}^{2} \\
\forall(v, \mu) \in H_{1 / 2}^{1}\left(\mathcal{T}_{h}\right) \times H_{0}^{-1 / 2}(\Gamma) .
\end{array}
$$

Next, let $\mathbf{A}_{h}$ be the semilinear form defined by the left-hand side of (6.12), i.e., $\mathbf{A}_{h}(w, \eta ; v, \mu):=A_{h}(w, v)+\langle\mathcal{W} w, v\rangle-\left\langle\left(\frac{1}{2} \mathcal{I}-\mathcal{K}^{\prime}\right) \eta, v\right\rangle+\left\langle\mu,\left(\frac{1}{2} \mathcal{I}-\mathcal{K}\right) w\right\rangle+\langle\mu, \mathcal{V} \eta\rangle$ 
for any $(w, \eta),(v, \mu) \in H_{1 / 2}^{1}\left(\mathcal{T}_{h}\right) \times H_{0}^{-1 / 2}(\Gamma)$. The following result shows that $\mathbf{A}_{h}$ is Lipschitz continuous and strongly monotone with respect to $\|\cdot\|_{h, \Gamma}$. This is crucial for the analysis of (6.12) (and hence of (6.8)).

Lemma 6.2. There exist positive constants $C_{L M}$ and $C_{S M}$, independent of $h$, such that

$$
\left|\mathbf{A}_{h}(w, \eta ; z, \xi)-\mathbf{A}_{h}(v, \mu ; z, \xi)\right| \leq C_{L M}\|(w, \eta)-(v, \mu)\|_{h, \Gamma}\|(z, \xi)\|_{h, \Gamma}
$$

and

(6.14)

$$
\mathbf{A}_{h}(w, \eta ;(w, \eta)-(v, \mu))-\mathbf{A}_{h}(v, \mu ;(w, \eta)-(v, \mu)) \geq C_{S M}\|(w, \eta)-(v, \mu)\|_{h, \Gamma}^{2}
$$

for any $(w, \eta),(v, \mu),(z, \xi) \in H_{1 / 2}^{1}\left(\mathcal{T}_{h}\right) \times H_{0}^{-1 / 2}(\Gamma)$.

Proof. The Lipschitz continuity and strong monotonicity of the semilinear form $A_{h}$ with respect to the norm $\|\cdot\|_{h}$ are provided by Lemmas 4.1 and 4.2 in [7]. The estimates required for the remaining boundary integral terms of $\mathbf{A}_{h}$ follow exactly as in the proof of Lemma 3.1. We omit further details.

The unique solvability of (6.8) is now established.

Theorem 6.1. There exists a unique $\left(\boldsymbol{\theta}_{h}, \boldsymbol{\sigma}_{h}, u_{h}, \lambda_{h}\right) \in \boldsymbol{\Sigma}_{h} \times \boldsymbol{\Sigma}_{h} \times \tilde{V}_{h} \times X_{h}^{0}$ solution to the coupled LDG-BEM scheme (6.8). In addition, there exists $C>0$, independent of $h$, such that

$$
\left\|\boldsymbol{\theta}_{h}\right\|_{0, \Omega}+\left\|\boldsymbol{\sigma}_{h}\right\|_{0, \Omega}+\left\|\left(u_{h}, \lambda_{h}\right)\right\|_{h, \Gamma} \leq C\left\{\mathcal{N}\left(f, g_{0}, g_{1}, g_{2}\right)+\|\overline{\mathbf{a}}(\cdot, 0)\|_{0, \Omega}\right\}
$$

where

$\mathcal{N}\left(f, g_{0}, g_{1}, g_{2}\right):=\left\{\|f\|_{0, \Omega}^{2}+\left\|\alpha^{1 / 2} g_{0}\right\|_{0, \Gamma_{0}}^{2}+\left\|\alpha^{1 / 2} g_{1}\right\|_{0, \Gamma_{1}}^{2}+\left\|\alpha^{1 / 2} g_{2}\right\|_{0, \Gamma_{1}}^{2}\right\}^{1 / 2}$.

Proof. By Lemma 6.1 it suffices to analyze the reduced system (6.12) instead of (6.8). It is clear that (6.12) can be equivalently formulated as: Find $\left(u_{h}, \lambda_{h}\right) \in$ $\tilde{V}_{h} \times X_{h}^{0}$ such that

$$
\mathbf{A}_{h}\left(u_{h}, \lambda_{h} ; v, \mu\right):=F_{h}(v) \quad \forall(v, \mu) \in \tilde{V}_{h} \times X_{h}^{0} .
$$

Now, proceeding as in the proof of Lemma 4.4 in [7], we find $C>0$, independent of $h$, such that

$$
\left|F_{h}(v)\right| \leq C \mathcal{N}\left(f, g_{0}, g_{1}, g_{2}\right)\|v\|_{h}, \quad \forall v \in \tilde{V}_{h} .
$$

Hence, Lemma 6.2 and a classical result of nonlinear functional analysis imply the unique solvability of (6.12). The rest of the proof follows very closely the proof of Theorem 3.2 in 9 . In fact, using again the strong monotonicity of $\mathbf{A}_{h}$, estimate (6.16), the fact that

$$
\mathbf{A}_{h}((0,0),(v, \mu))=A_{h}(0, v)=\int_{\Omega} \overline{\mathbf{a}}\left(\cdot, \mathcal{G}_{h}\right) \cdot\left(\nabla_{h} v-\boldsymbol{S}_{h} v\right) \quad \forall(v, \mu) \in \tilde{V}_{h} \times X_{h}^{0},
$$

the boundedness of $\mathbf{S}_{h}$ (cf. (3.6) ), and the Lipschitz continuity of the nonlinear operator induced by $\overline{\mathbf{a}}$, one deduces that

$$
\left\|\left(u_{h}, \lambda_{h}\right)\right\|_{h, \Gamma} \leq C\left\{\mathcal{N}\left(f, g_{0}, g_{1}, g_{2}\right)+\|\overline{\mathbf{a}}(\cdot, 0)\|_{0, \Omega}+\left\|\mathcal{G}_{h}\right\|_{0, \Omega}\right\} .
$$


Also, using the expressions for $\boldsymbol{\theta}_{h}$ and $\boldsymbol{\sigma}_{h}$ given by (6.11), and applying again the boundedness of $\mathbf{S}_{h}$ and the Lipschitz continuity of $\overline{\mathbf{a}}$, we obtain

$\left\|\boldsymbol{\theta}_{h}\right\|_{0, \Omega} \leq C\left\{\left\|u_{h}\right\|_{h}+\left\|\mathcal{G}_{h}\right\|_{0, \Omega}\right\} \quad$ and $\quad\left\|\boldsymbol{\sigma}_{h}\right\|_{0, \Omega} \leq C\left\{\left\|\boldsymbol{\theta}_{h}\right\|_{0, \Omega}+\|\overline{\mathbf{a}}(\cdot, 0)\|_{0, \Omega}\right\}$.

Then, it is easy to show, as in the proof of Lemma 3.4 in [7, that (cf. (6.10)

$$
\left\|\mathcal{G}_{h}\right\|_{0, \Omega} \leq C\left\{\left\|\alpha^{1 / 2} g_{0}\right\|_{0, \Gamma_{0}}+\left\|\alpha^{1 / 2} g_{1}\right\|_{0, \Gamma_{1}}\right\} .
$$

In this way, (6.15) follows directly from (6.17), (6.18), and (6.19), which ends the proof.

Finally, we prove the a priori error estimate for the coupled LDG-BEM scheme (6.8).

Theorem 6.2. Define the additional continuous unknowns

$\boldsymbol{\theta}=\left\{\begin{array}{l}\boldsymbol{\theta}_{1}:=\nabla u_{1} \text { in } \Omega_{1}, \\ \boldsymbol{\theta}_{2}:=\nabla u_{2} \text { in } \Omega_{2},\end{array} \quad \boldsymbol{\sigma}=\left\{\begin{array}{l}\boldsymbol{\sigma}_{1}:=\mathbf{a}\left(\cdot, \boldsymbol{\theta}_{1}\right) \text { in } \Omega_{1}, \\ \boldsymbol{\sigma}_{2}:=\boldsymbol{\theta}_{2} \text { in } \Omega_{2},\end{array}\right.\right.$ and $\lambda=\partial_{\boldsymbol{\nu}} u_{2}$ on $\Gamma$.

Assume that there exist $\delta_{1}, \delta_{2}>1 / 2$ such that $u_{1} \in H^{1+\delta_{1}}\left(\Omega_{1}\right), u_{2} \in H^{1+\delta_{2}}\left(\Omega_{2}\right)$, and $\sigma_{1} \in\left[H^{\delta_{1}}\left(\Omega_{1}\right)\right]^{2}$. Then there exists $C>0$, independent of $h$, such that

$$
\begin{aligned}
\left\|\boldsymbol{\theta}-\boldsymbol{\theta}_{h}\right\|_{0, \Omega}+\left\|\boldsymbol{\sigma}-\boldsymbol{\sigma}_{h}\right\|_{0, \Omega}+ & \left\|(u, \lambda)-\left(u_{h}, \lambda_{h}\right)\right\|_{h, \Gamma} \\
\leq C\left\{h^{\min \left\{\delta_{1}, m\right\}}\left\|u_{1}\right\|_{1+\delta_{1}, \Omega_{1}}+\right. & h^{\min \left\{\delta_{1}, m\right\}}\left\|\boldsymbol{\sigma}_{1}\right\|_{\delta_{1}, \Omega_{1}} \\
& \left.+h^{\min \left\{\delta_{2}, m\right\}}\left\|u_{2}\right\|_{1+\delta_{2}, \Omega_{2}}\right\} .
\end{aligned}
$$

Proof. We observe, similarly as in the linear case (cf. Theorem 4.1), that $\lambda \in$ $H^{\delta_{2}-1 / 2}(\Gamma)$ and $\|\lambda\|_{\delta_{2}-1 / 2, \Gamma} \leq C\left\|u_{2}\right\|_{1+\delta_{2}, \Omega_{2}}$. Also, according to the definitions of the semilinear form $\mathbf{A}_{h}$ and the linear operator $F_{h}$, and taking into account the equations, the boundary conditions, and the transmission conditions satisfied by $u$, one can prove that $u$ and $\lambda$ satisfy

$$
\mathbf{A}_{h}(u, \lambda ; v, \mu)=F_{h}(v) \quad \forall(v, \mu) \in H_{1 / 2}^{1}\left(\mathcal{T}_{h}\right) \times H^{-1 / 2}(\Gamma) .
$$

In addition, it is clear that the discrete system (6.12) renders

$$
\mathbf{A}_{h}\left(u_{h}, \lambda_{h} ; v, \mu\right)=F_{h}(v) \quad \forall(v, \mu) \in \tilde{V}_{h} \times X_{h}^{0} .
$$

Then, the Lipschitz continuity and strong monotonicity of $\mathbf{A}_{h}$ also yield the quasioptimal estimate (4.15); that is,

$$
\left\|(u, \lambda)-\left(u_{h}, \lambda_{h}\right)\right\|_{h, \Gamma} \leq C\left\|(u, \lambda)-\left(v_{h}, \mu_{h}\right)\right\|_{h, \Gamma} \quad \forall\left(v_{h}, \mu_{h}\right) \in \tilde{V}_{h} \times X_{h}^{0} .
$$

Now, using that $\boldsymbol{\theta}_{h}=\nabla_{h} u_{h}-\mathbf{S}_{h}\left(u_{h}\right)+\mathcal{G}_{h}$ (cf. (6.11)), $\boldsymbol{\theta}=\nabla u$ in $\Omega, \mathbf{S}_{h}(u)=\mathcal{G}_{h}$, and applying the boundedness of $\mathbf{S}_{h}$, we obtain

$$
\left\|\boldsymbol{\theta}-\boldsymbol{\theta}_{h}\right\|_{0, \Omega} \leq C\left\|u-u_{h}\right\|_{h} .
$$

It remains to estimate $\left\|\boldsymbol{\sigma}-\boldsymbol{\sigma}_{h}\right\|_{0, \Omega}$. Using that $\boldsymbol{\sigma}_{h}=\Pi_{\boldsymbol{\Sigma}_{h}} \overline{\mathbf{a}}\left(\cdot, \boldsymbol{\theta}_{h}\right)$ (cf. (6.11)) and $\boldsymbol{\sigma}=\overline{\mathbf{a}}(\cdot, \boldsymbol{\theta})$, and applying the triangle inequality and the Lipschitz continuity of the nonlinear operator induced by $\overline{\mathbf{a}}$, we deduce that

$$
\begin{aligned}
\left\|\boldsymbol{\sigma}-\boldsymbol{\sigma}_{h}\right\|_{0, \Omega} & \leq\left\|\boldsymbol{\sigma}-\Pi_{\boldsymbol{\Sigma}_{h}} \boldsymbol{\sigma}\right\|_{0, \Omega}+\left\|\Pi_{\boldsymbol{\Sigma}_{h}}\left\{\overline{\mathbf{a}}(\cdot, \boldsymbol{\theta})-\overline{\mathbf{a}}\left(\cdot, \boldsymbol{\theta}_{h}\right)\right\}\right\|_{0, \Omega} \\
& \leq\left\|\boldsymbol{\sigma}-\Pi_{\boldsymbol{\Sigma}_{h}} \boldsymbol{\sigma}\right\|_{0, \Omega}+C\left\|\boldsymbol{\theta}-\boldsymbol{\theta}_{h}\right\|_{0, \Omega} .
\end{aligned}
$$


Then, applying local approximation properties of piecewise polynomials (see, e.g. Lemma 4.2 in [18]), recalling from (2.6) that on $K \in \mathcal{T}_{h}, \Pi_{\boldsymbol{\Sigma}_{h}}$ reduces to the $\mathbf{L}^{2}(K)$-orthogonal projection onto $\mathbf{P}_{r}(K)$, which is denoted by $\Pi_{K}^{r}$, and noting that $r+1 \geq m$, we find that

$$
\begin{aligned}
\left\|\boldsymbol{\sigma}-\Pi_{\boldsymbol{\Sigma}_{h}} \boldsymbol{\sigma}\right\|_{0, \Omega_{1}} & =\sum_{K \in \mathcal{T}_{h, 1}}\left\|\boldsymbol{\sigma}_{1}-\Pi_{K}^{r} \boldsymbol{\sigma}_{1}\right\|_{0, K}^{2} \\
& \leq C \sum_{K \in \mathcal{T}_{h, 1}} h_{K}^{2 \min \left\{\delta_{1}, r+1\right\}}\left\|\boldsymbol{\sigma}_{1}\right\|_{\delta_{1}, K}^{2} \\
& \leq C h^{2 \min \left\{\delta_{1}, r+1\right\}}\left\|\boldsymbol{\sigma}_{1}\right\|_{\delta_{1}, \Omega_{1}}^{2} \leq C h^{2 \min \left\{\delta_{1}, m\right\}}\left\|\boldsymbol{\sigma}_{1}\right\|_{\delta_{1}, \Omega_{1}}^{2}
\end{aligned}
$$

and

$$
\begin{aligned}
\| \boldsymbol{\sigma} & -\Pi_{\boldsymbol{\Sigma}_{h}} \boldsymbol{\sigma}\left\|_{0, \Omega_{2}}^{2}=\sum_{K \in \mathcal{T}_{h, 2}}\right\| \boldsymbol{\theta}_{2}-\Pi_{K}^{r} \boldsymbol{\theta}_{2} \|_{0, K}^{2} \\
& =\sum_{K \in \mathcal{T}_{h, 2}}\left\|\nabla u_{2}-\Pi_{K}^{r} \nabla u_{2}\right\|_{0, K}^{2} \leq C \sum_{K \in \mathcal{T}_{h, 2}} h_{K}^{2 \min \left\{\delta_{2}, r+1\right\}}\left\|\nabla u_{2}\right\|_{\delta_{2}, K}^{2} \\
& \leq C h^{2 \min \left\{\delta_{2}, r+1\right\}}\left\|u_{2}\right\|_{1+\delta_{2}, \Omega_{2}}^{2} \leq C h^{2 \min \left\{\delta_{2}, m\right\}}\left\|u_{2}\right\|_{1+\delta_{2}, \Omega_{2}}^{2} .
\end{aligned}
$$

In this way, the approximation properties from Lemmas 4.2 and 4.3 (with $t=$ $\left.\delta_{2}-1 / 2\right)$, together with the bound $\|\lambda\|_{\delta_{2}-1 / 2, \Gamma} \leq C\left\|u_{2}\right\|_{1+\delta_{2}, \Omega_{2}}$, and inequalities (6.21), (6.22), 6.23), (6.24), and (6.25), imply the required a priori error estimate and finish the proof.

We end this section by remarking, as we did for the linear case at the end of Section 4, that the a priori error estimate (6.20) is also independent of the polynomial degree $r$ that defines the subspace $\boldsymbol{\Sigma}_{h}$ (cf. (2.6)). Therefore, since the restriction $r \geq m-1$ is required only to deduce that $\boldsymbol{\theta}_{h}=\nabla_{h} u_{h}-\mathbf{S}_{h}\left(u_{h}\right)+\mathcal{G}_{h}$ (cf. (6.11)), it suffices also to take $r=m-1$ in the present nonlinear case.

\section{Coupling with other DG methods}

As exposed, the theory on coupling the LDG method with a symmetric boundary element formulation demanding continuity on the trace for the discontinuous function can be extended to several other DG methods. We give here some very fast brushstrokes for three methods, all of which can be introduced more naturally in the primal form, i.e., expressed directly on the variable $u_{h}$, which is precisely the way we have approached the analysis. We remark, however, that for the extension to nonlinear problems given in the previous section, the formulation with two variables in the domain (the one with mixed flavor) has to be used.

The simplest adaptation is given by the method with discontinuous polynomials and jump penalization on the element interfaces, that can be traced back to Babuška and Zlámal (see [5]). In our notation it consists simply of erasing completely the bilinear form $S$ or equivalently the gradient correction term $\mathbf{S}_{h}$ in all occurrences. In this case $\boldsymbol{\sigma}_{h}$ is simply $\nabla_{h} u_{h}$. The expression of the bilinear form $B_{h}$ (see (2.16)) is greatly simplified and everything that has been said for this bilinear form (i.e., (3.4), (3.5) and (4.14)) still applies, so we can carry out our analysis to the very end. 
The Interior Penalty method [15, 1] consists of taking the bilinear form

$$
\begin{aligned}
B_{h}(u, v):=\boldsymbol{\alpha}(u, v)+\int_{\Omega} \nabla_{h} u \cdot \nabla_{h} v-\int_{I_{h}}\left(\llbracket u \rrbracket \cdot\left\{\nabla_{h} v\right\}\right. & \left.+\llbracket v \rrbracket \cdot\left\{\nabla_{h} u\right\}\right) \\
& -\int_{\Gamma_{0}}\left(u \partial_{\boldsymbol{\nu}} v+v \partial_{\boldsymbol{\nu}} u\right)
\end{aligned}
$$

and go on as usual. This is equivalent to taking $\boldsymbol{\beta}=0$ in the LDG method and subtracting a term

$$
\int_{\Omega} \mathbf{S}_{h}(u) \cdot \mathbf{S}_{h}(v)
$$

from the bilinear form $B_{h}$. The corresponding flux is $\boldsymbol{\sigma}_{h}=\nabla_{h} u_{h}-\mathbf{S}_{h}\left(u_{h}\right)$. It is possible to unfold the system to write it in the expanded form reminiscent of (2.12). The difference is the fact that the second equation

$$
\int_{\Omega} \nabla_{h} v \cdot \boldsymbol{\sigma}_{h}-S\left(v, \boldsymbol{\sigma}_{h}\right)+\boldsymbol{\alpha}\left(u_{h}, v\right)+\left\langle\mathcal{W} u_{h}, v\right\rangle-\left\langle\left(\frac{1}{2} \mathcal{I}-\mathcal{K}^{\prime}\right) \lambda_{h}, v\right\rangle=\int_{\Omega} f v
$$

is substituted by

$$
\int_{\Omega} \nabla_{h} v \cdot \boldsymbol{\sigma}_{h}-S\left(v, \nabla_{h} u_{h}\right)+\boldsymbol{\alpha}\left(u_{h}, v\right)+\left\langle\mathcal{W} u_{h}, v\right\rangle-\left\langle\left(\frac{1}{2} \mathcal{I}-\mathcal{K}^{\prime}\right) \lambda_{h}, v\right\rangle=\int_{\Omega} f v .
$$

The global system loses in this way its symmetry (the symmetry of (2.12) is only apparent after changing the sign of the second equation) in this unfolded form, a symmetry that is recovered once the variable $\boldsymbol{\sigma}_{h}$ has been eliminated from the system. The only intricate part of the analysis refers to recovering the discrete ellipticity (3.5), which imposes some restrictions on the function that defines the bilinear form $\boldsymbol{\alpha}$; see 2 for the details. Apart from this, the remaining part of our analysis can be carried out without much difficulty.

Last but not least, NIPG also fits easily in this framework. The bilinear form in the primal formulation is

$$
\begin{aligned}
B_{h}(u, v):=\boldsymbol{\alpha}(u, v)+\int_{\Omega} \nabla_{h} u \cdot \nabla_{h} v & -\int_{I_{h}}\left(\llbracket u \rrbracket \cdot\left\{\nabla_{h} v\right\}-\llbracket v \rrbracket \cdot\left\{\nabla_{h} u\right\}\right) \\
& -\int_{\Gamma_{0}}\left(u \partial_{\boldsymbol{\nu}} v-v \partial_{\boldsymbol{\nu}} u\right),
\end{aligned}
$$

(i.e., desymmetrizes the interface terms of IP) and makes the ellipticity estimate (3.5) a simple consequence, since the corresponding quadratic form is the same as in the first method we have just exposed. As in the previous case, it is possible to add $\sigma_{h}$ as an unknown and unfold the system (cf. 2]).

\section{REFERENCES}

[1] D.N. Arnold, An interior penalty finite element method with discontinuous elements. SIAM Journal on Numerical Analysis, vol. 19, 4, pp. 742-760, (1982). MR664882 (83f:65173)

[2] D.N. Arnold, F. Brezzi, B. Cockburn and L.D. Marini, Unified analysis of discontinuous Galerkin methods for elliptic problems. SIAM Journal on Numerical Analysis, vol. 39, 5, pp. 1749-1779, (2002). MR1885715 (2002k:65183)

[3] I. Babuška and M. Suri, The h-p version of the finite element method with quasiuniform meshes. RAIRO Modélisation Mathématique et Analyse Numérique, vol. 21, pp. 199-238, (1987). MR896241 (88d:65154)

[4] I. Babuška and M. Suri, The optimal convergence rate of the p-version of the finite element method. SIAM Journal on Numerical Analysis, vol. 24, pp. 750-776, (1987). MR899702 (88k:65102) 
[5] I. Babuška and M. Zlámal, Nonconforming elements in the finite element method with penalty. SIAM Journal on Numerical Analysis, vol. 10, pp. 863-875, (1973). MR0345432 (49:10168)

[6] A. Bespalov and N. Heuer, The hp-version of the boundary element method with quasiuniform meshes in three dimensions. ESAIM Mathematical Modelling and Numerical Analysis, vol. 42, 5, pp. 821-849, (2008). MR2454624 (2009f:65277)

[7] R. Bustinza and G.N. Gatica, A local discontinuous Galerkin method for nonlinear diffusion problems with mixed boundary conditions. SIAM Journal on Scientific Computing, vol. 26, 1, pp. 152-177, (2004). MR2114338 (2005k:65201)

[8] R. Bustinza and G.N. Gatica, A mixed local discontinuous Galerkin method for a class of nonlinear problems in fluid mechanics. Journal of Computational Physics, vol. 207, pp. 427456, (2005). MR2144625 (2006a:76069)

[9] R. Bustinza, G.N. Gatica and F.-J. Sayas, On the coupling of local discontinuous Galerkin and boundary element methods for nonlinear exterior transmission problems. IMA Journal of Numerical Analysis, vol. 28, 2, pp. 225-244, (2008). MR2401197 (2009c:65295)

[10] R. Bustinza, G.N. Gatica and F.-J. Sayas, A LDG-BEM coupling for a class of nonlinear exterior transmission problems. In Numerical Mathematics and Advanced Applications: Proceedings of ENUMATH 2005 (A. Bermúdez de Castro, D. Gómez, P. Quintela, and P. Salgado, eds.), pp. 1129-1136, Springer-Verlag, 2006. MR.2303745

[11] R. Bustinza, G.N. Gatica and F.-J. Sayas, A look at how LDG and BEM can be coupled. ESAIM Proceedings, vol. 21, pp. 88-97, (2007). MR2404055 (2009c:65332)

[12] B. Cockburn and C. Dawson, Some extensions of the local discontinuous Galerkin method for convection-diffusion equations in multidimensions. In Proceedings of the 10th Conference on the Mathematics of Finite Elements and Applications, J. R. Whiteman, ed., Elsevier, 2000, pp. 225-238. MR.1801979 (2001j:65142)

[13] M. Costabel, Symmetric methods for the coupling of finite elements and boundary elements. In Boundary Elements IX, vol. 1 (C. A. Brebbia et al., eds.), pp. 411-420, Springer, 1987 MR965328 (89j:65068)

[14] M. Costabel, Boundary integral operators on Lipschitz domains: Elementary results. SIAM Journal on Mathematical Analysis, vol. 19, pp. 613-626, (1988). MR937473 (89h:35090)

[15] J. Douglas Jr., T. Dupont, Interior Penalty Procedures for Elliptic and Parabolic Galerkin Methods. Lecture notes in Physics, vol. 58, Springer, Berlin, 1976. MR0440955 (55:13823)

[16] M. Feistauer, Mathematical and numerical study of nonlinear problems in fluid mechanics. In Proc. Conf. Equadiff 6, edited by J. Vosmansky and M. Zlámal, Brno 1985, Springer, Berlin, pp. 3-16. MR877102 (88f:76002)

[17] M. Feistauer, On the finite element approximation of a cascade flow problem. Numerische Mathematik, vol. 50, pp. 655-684, (1997). MR884294 (88h:65205)

[18] G.N. Gatica and F.-J. Sayas, An a priori error analysis for the coupling of local discontinuous Galerkin and boundary element methods. Mathematics of Computation, vol. 75, pp. 16751696, (2006). MR2240630 (2007e:65119)

[19] P. Grisvard, Singularities in Boundary Value Problems. Recherches in Mathématiques Appliquées, Masson, Paris, 1992. MR.1173209 (93h:35004)

[20] H. Han, A new class of variational formulations for the coupling of finite and boundary element methods. Journal of Computational Mathematics, vol. 8, 3, pp. 223-232, (1990). MR.1299224

[21] B. Heise, Nonlinear field calculations with multigrid Newton methods. Impact of Computing in Science and Engineering, vol. 5, pp. 75-110, (1993). MR.1223880 (95a:78002)

[22] B. Heise, Analysis of a fully discrete finite element method for a nonlinear magnetic field problem. SIAM Journal on Numerical Analysis, vol. 31, 3, pp. 745-759, (1994). MR1275111 (95i:65156)

[23] P. Houston, J. Robson and E. Süli, Discontinuous Galerkin finite element approximation of quasilinear elliptic boundary value problems I: the scalar case. IMA Journal of Numerical Analysis, vol. 25, 4, pp. 726-749, (2005). MR2170521 (2006k:65322)

[24] G.C. Hsiao and W. Wendland, Boundary Integral Equations. Applied Mathematical Sciences, vol. 164, Springer-Verlag Berlin Heidelberg, 2008. MR.2441884 (2009i:45001)

[25] J.-C. Nédélec, Integral equations with nonintegrable kernels. Integral Equations and Operator Theory, vol. 5, pp. 562-572, (1982). MR665149 (84i:45011) 
[26] B. Rivière, M.F. Wheeler and V. Girault, Improved energy estimates for interior penalty, constrained and discontinuous Galerkin methods for elliptic problems I. Computational Geosciences, vol. 3, pp. 337-360, (1999). MR.1750076 (2001d:65145)

CI²Ma and Departamento de Ingeniería Matemática, Universidad de Concepción, Casilla 160-C, Concepción, Chile

E-mail address: ggatica@ing-mat.udec.cl

Facultad de Matemáticas, Pontificia Universidad Católica de Chile, Casilla 306, Correo 22, Santiago, Chile,

E-mail address: nheuer@mat.puc.cl

Departamento de Matemática Aplicada, Centro Politécnico Superior, Universidad De Zaragoza, María de Luna, 3 - 50018 Zaragoza, Spain

E-mail address: jsayas@unizar.es

Current address: School of Mathematics, University of Minnesota, 206 Church St. SE, Minneapolis, Minnesota 55455 USA 